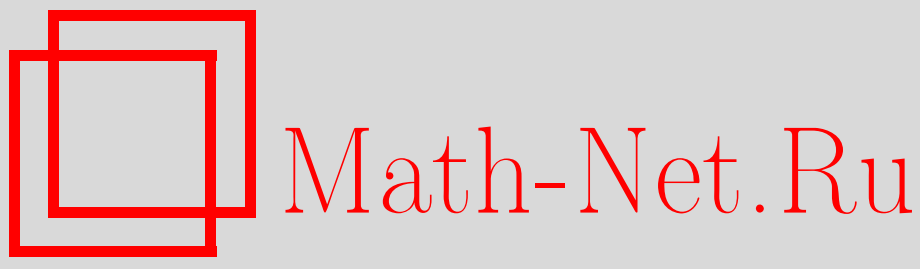

В. И. Богачев, М. Рёкнер, С. В. Шапошников, Положительные плотности переходных вероятностей диффузионных процессов, Теория вероятн. и ее примен., 2008, том 53, выпуск 2, 213-239

DOI: https://doi.org/10.4213/tvp1725

Использование Общероссийского математического портала Math-Net.Ru подразумевает, что вы прочитали и согласны с пользовательским соглашением

http://www . mathnet.ru/rus/agreement

Параметры загрузки:

IP: 34.229 .108 .108

26 апреля 2023 г., $10: 34: 23$

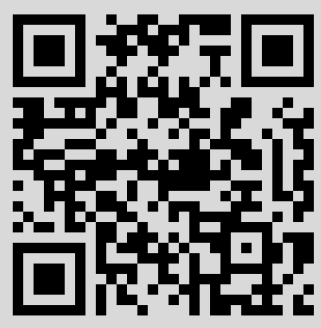




\section{ПОЛОЖИТЕЛЬНЫЕ ПЛОТНОСТИ ПЕРЕХОДНЫХ ВЕРОЯТНОСТЕЙ ДИФФУЗИОННЫХ ПРОЦЕССОВ ${ }^{1)}$}

Для диффузионных процессов в $\mathbf{R}^{d}$ с локально неограниченными коэффициентами сноса получено достаточное условие строгой положительности переходных вероятностей. Для этого рассматриваются параболические уравнения вида $\mathscr{L}^{*} \mu=0$ относительно мер на $\mathbf{R}^{d} \times(0,1)$ с оператором

$$
\mathscr{L} u:=\partial_{t} u+\partial_{x_{i}}\left(a^{i j} \partial_{x_{j}} u\right)+b^{i} \partial_{x_{i}} u .
$$

Показано, что если коэффициент диффузии $A=\left(a^{i j}\right)$ достаточно регулярен, а коэффициент сноса $b=\left(b^{i}\right)$ удовлетворяет условию $\exp \left(\kappa|b|^{2}\right) \in L_{\text {loc }}^{1}(\mu)$, причем мера $\mu$ неотрицательна, то $\mu$ обладает непрерывной плотностью $\varrho(x, t)$, которая строго положительна при $t>\tau$, если она не равна нулю тождественно при $t \leqslant \tau$. Получены применения к конечномерным проекциям стационарных распределений и переходных вероятностей бесконечномерных диффузий.

Ключевые слова и фразы: плотность переходной вероятности, стационарное распределение, параболическое уравнение, бесконечномерная диффузия.

1. Введение. В работе исследуются условия строгой положительности плотностей переходных вероятностей конечномерных диффузионных процессов с сингулярными коэффициентами сноса, а также строгой положительности и непрерывности плотностей конечномерных проекций стационарных распределений и переходных вероятностей бесконечномерных диффузий. Для этого мы рассматриваем уравнения вида

$$
\mathscr{L}^{*} \mu=0
$$

* Московский государственный университет им. М.В. Ломоносова, механикоматематический факультет, Ленинские горы, 119992 Москва, Россия.

** Fakultät für Mathematik, Universität Bielefeld, D-33501 Bielefeld, Germany; Department of Mathematics, Purdue University, 150 N. University Str., West Lafayette, IN 47907-2067, USA.

1) Работа выполнена при поддержке проектов РФФИ 07-01-00536, 08-01-91205-ЯФ и 06-01-39003 ГФЕН, НШ 1758.2003.1, ARC Discovery Grant DP0663153, DFG 436 RUS 113/343/0(R) и SFB 701 при университете Билефельда. 
относительно борелевских мер $\mu$ на $\mathbf{R}^{d}$ или на $\mathbf{R}^{d} \times(0,1)$. Здесь $\mathscr{L}-$ эллиптический или параболический оператор второго порядка вида

$$
\mathscr{L} u(x):=\partial_{x_{i}}\left(a^{i j} \partial_{x_{j}} u(x)\right)+b^{i}(x) \partial_{x_{i}} u(x)
$$

или

$$
\mathscr{L} u(x, t):=\partial_{t} u(x, t)+\partial_{x_{i}}\left(a^{i j}(x, t) \partial_{x_{j}} u(x, t)\right)+b^{i}(x, t) \partial_{x_{i}} u(x, t),
$$

где ведется суммирование по повторяющимся индексам, а интерпретация нашего уравнения состоит в следуюшем.

Будем говорить, что борелевская мера $\mu$ на $\mathbf{R}^{d}$ удовлетворяет слабому эллиптическому уравнению (1.1), если функции $a^{i j}$ входят в локальный соболевский класс $W_{\mathrm{loc}}^{1,1}\left(\mathbf{R}^{d}\right)$, функции $a^{i j}, \partial_{x_{i}} a^{i j}$ и $b^{i}$ борелевски измеримы и локально интегрируемы относительно $|\mu|$, причем для каждой функции $u \in C_{0}^{\infty}\left(\mathbf{R}^{d}\right)$ выполнено равенство

$$
\int_{\mathbf{R}^{d}}\left[a^{i j} \partial_{x_{i}} \partial_{x_{j}} u+\partial_{x_{i}} a^{i j} \partial_{x_{j}} u+b^{i} \partial_{x_{i}} u\right] d \mu=0 .
$$

Аналогично, борелевская мера $\mu$ на $\mathbf{R}^{d} \times(0,1)$ удовлетворяет слабому параболическому уравнению (1.1), если для каждого отрезка $J \subset(0,1)$ и каждого шара $U \subset \mathbf{R}^{d}$ функции $a^{i j}$ принадлежат определяемому ниже классу $\mathbf{H}^{1,1}(U, J)$, функции $a^{i j}, \partial_{x_{i}} a^{i j}$ и $b^{i}$ борелевски измеримы и локально интегрируемы относительно $|\mu|$, причем для каждой функции $u \in C_{0}^{\infty}\left(\mathbf{R}^{d} \times(0,1)\right)$ выполнено равенство

$$
\int_{\mathbf{R}^{d} \times(0,1)}\left[\partial_{t} u+a^{i j} \partial_{x_{i}} \partial_{x_{j}} u+\partial_{x_{i}} a^{i j} \partial_{x_{j}} u+b^{i} \partial_{x_{i}} u\right] d \mu=0 .
$$

Аналогичным образом уравнение (1.1) определяется для оператора $\mathscr{L}$ с дополнительным членом $\theta \cdot u$, где функция $\theta$ локально $\mu$-интегрируема.

Всюду ниже предполагается, что $a^{i j}=a^{j i}$ и что матрицы $A(x, t)=$ $\left(a^{i j}(x, t)\right)$ положительны. Векторное поле $b=\left(b^{1}, \ldots, b^{d}\right)$ называется коэффициентом сноса и $A$ называется коэффициентом диффузии.

Таким уравнениям при весьма широких условиях удовлетворяют стационарные распределения и переходные вероятности диффузионных процессов. Если диффузионный процесс $\xi_{t}$ в $\mathbf{R}^{d}$ задан стохастическим дифференциальным уравнением в форме Ито

$$
d \xi_{t}=\sqrt{A\left(\xi_{t}, t\right)} d W_{t}+\frac{1}{2} b\left(\xi_{t}, t\right) d t
$$

где $W_{t}$ - винеровский процесс в $\mathbf{R}^{d}$, то переходные вероятности $P(t, B):=\mathbf{P}\left\{\xi_{t} \in B\right\}$ процесса $\xi_{t}$ (или $P(s, z ; t, B):=\mathbf{P}\left\{\xi_{t} \in B \mid \xi_{s}=z\right\}$ с фиксированными $s \in \mathbf{R}^{1}$ и $z \in \mathbf{R}^{d}$ ) задают меру $\mu=P(t, d x) d t$ 
на $\mathbf{R}^{d} \times(0,1)$, удовлетворяющую параболическому уравнению $L^{*} \mu=0$ в смысле аналогичного (1.5) тождества, но с оператором $L$ недивергентного вида

$$
L u(x, t):=\partial_{t} u(x, t)+a^{i j}(x, t) \partial_{x_{i}} \partial_{x_{j}} u(x, t)+b^{i}(x, t) \partial_{x_{i}} u(x, t),
$$

а в случае, когда коэффициенты не зависят от времени и существует стационарное распределение $\mu$, для этого распределения выполнено эллиптическое уравнение $L^{*} \mu=0$ в смысле аналогичного (1.4) тождества также с оператором $L$ недивергентного вида

$$
L u(x):=a^{i j} \partial_{x_{i}} \partial_{x_{j}} u(x)+b^{i}(x) \partial_{x_{i}} u(x) .
$$

Уравнения с операторами дивергентного вида (1.2) и (1.3) возникают для стохастических уравнений в форме Стратоновича. Однако при наших условиях относительно $a^{i j}$ недивергентный оператор $L$ очевидным образом записывается в дивергентном виде (1.2) или (1.3) с новым сносом с компонентами $b^{j}-\partial_{x_{i}} a^{i j}$. Поэтому все результаты настоящей работы верны и для недивергентных операторов (1.6) и 1.7) и, тем самым, применимы к стохастическим уравнениям в форме Ито, если соответствующие условия на снос выполнены для указанным образом измененного сноса. Мы рассматриваем операторы дивергентного вида лишь по той причине, что это приводит к некоторым техническим удобствам с точки зрения параболических уравнений.

Хорошо известно, что в случае достаточно регулярных коэффициентов $A$ и $b$ и невырожденности $A$ переходные вероятности и стационарные распределения обладают непрерывными строго положительными плотностями; строгая положительность обычно выводится из неравенства Харнака. В работе [1] было показано, что если коэффициенты $b^{i}$ локально интегрируемы по Лебегу в достаточно высокой степени (больше $d$ в эллиптическом случае и больше $d+2$ в параболическом случае), а коэффициент диффузии $A$ невырожден и достаточно регулярен, то $\mu$ допускает непрерывную строго положительную плотность. Однако имеются важные приложения, в которых интегрируемость $b$ дана лишь относительно $\mu$; типичное приложение связано с конечномерномерными проекциями бесконечномерных диффузий, что обсуждается ниже. Другим типичным примером является мера $\mu$ с гладкой плотностью $\varrho$, которая удовлетворяет эллиптическому уравнению (1.1) с

$$
A(x)=\mathrm{Id} \quad \text { и } \quad b(x)=\frac{\nabla \varrho(x)}{\varrho(x)},
$$

где $b(x):=0$ при $\varrho(x)=0$. Действительно, соответствующее интегральное соотношение принимает вид

$$
\int_{\mathbf{R}^{d}}[\Delta u(x) \varrho(x)+(\nabla \varrho(x), \nabla u(x))] d x=0,
$$


что выполнено для всех $u \in C_{0}^{\infty}\left(\mathbf{R}^{d}\right)$ ввиду формулы интегрирования по частям. Если $\varrho$ обращается в нуль (в некоторых точках или даже на некоторых множествах с непустой внутренностью), то поле $b=\nabla \varrho / \varrho$ может быть весьма сингулярным с точки зрения меры Лебега, хотя и локально интегрируемым относительно $\mu$. В этом случае непрерывная плотность $\mu$ может обращаться в нуль даже при весьма высокой интегрируемости $b$ относительно $\mu$. Например, мера $\mu$ на вешественной прямой с плотностью $\varrho$ такой, что $\varrho(x)=\exp \left(-x^{-2}\right)$ при $x>0$ и $\varrho(x)=0$ при $x \leqslant 0$, удовлетворяет уравнению (1.1) с $A=1$ и $b(x)=2 x^{-3}$. Здесь $b \in L^{p}(\mu)$ для всех $p \in[1,+\infty)$. Однако для эллиптического случая в работе [2] было показано, что $\mu$-интегрируемость $\exp (\kappa|b|)$ при некотором $\kappa>0$ дает строгую положительность плотности меры $\mu$. Здесь мы докажем параболический аналог этого результата, утверждающий, что при разумных предположениях относительно коэффициента $A$ локальная $\mu$-интегрируемость $\exp \left(\kappa|b|^{2}\right)$ при некотором $\kappa>0$ обеспечивает существование непрерывной плотности $\varrho$ меры $\mu$, для которой $\varrho(x, t)>0$ при $t>\tau$, если $\varrho(x, t)$ не равняется нулю тождественно при $t \leqslant \tau$. Применительно к плотностям переходных вероятностей это дает строгую положительность при всех $t>0$. В отличие от эллиптического случая, нельзя заменить $|b|^{2}$ на $|b|$, что демонстрируется с помошью следующего простого примера. Положим $d=1$,

$$
b(x, t)=-\varepsilon(t-a)^{-2} e^{\varepsilon x}+2 \varepsilon^{-1}(t-a)^{-1},
$$

где $\varepsilon, a \in(0,1)$, причем $\mathscr{L} f(x, t)=\partial_{t} f(x, t)+\partial_{x}^{2} f(x, t)+b(x, t) \partial_{x} f(x, t)$. Тогда мера

$$
\mu=\exp \left(-(t-a)^{-2} e^{\varepsilon x}\right) d x d t
$$

удовлетворяет уравнению $\mathscr{L}^{*} \mu=0$ на $\mathbf{R}^{1} \times(0,1)$, но при $t=a$ ее непрерывная плотность равна нулю. Легко видеть, что функция $\exp (\kappa|b|)$ интегрируема относительно $\mu$, если $\varepsilon<\kappa^{-1}$. В качестве приложения в последнем пункте мы получим достаточные условия на коэффициенты бесконечномерной диффузии $\xi_{t}$ со сносом $b(x, t)$, гарантирующие, что конечномерные проекции стационарных распределений и переходных вероятностей обладают строго положительными плотностями. Например, если коэффициент диффузии процесса $\xi_{t}$ в $\mathbf{R}^{\infty}$ постоянен и невырожден и $b=\left(b^{i}\right)$, то для сушествования строго положительных непрерывных плотностей всех конечномерных проекций переходных вероятностей $P_{t}(x, \cdot)$ достаточно, чтобы функции $\exp \left(\kappa\left|b^{i}\right|^{2}\right)$ были интегрируемы относительно меры $P_{t}(x, \cdot) d t$. Для диффузии в банаховом пространстве с коэффициентом диффузии Id и сносом $b$ достаточно иметь оценку $\|b(x, t)\| \leqslant C+C\|x\|$.

Отметим, что в работах [3]-[6] можно найти верхние и нижние оценки плотностей решений. Вопросы сушествования решений уравне- 
ний указанного типа рассмотрены в [7], [8], а проблемам единственности посвящены работы [9]-[11].

Для произвольной области $\Omega \subset \mathbf{R}^{d}$ через $W^{p, 1}(\Omega)$ обозначим пространство Соболева всех функций, принадлежацих $L^{p}(\Omega)$ вместе с их обобщенными частными производными первого порядка. Это пространство наделено стандартной нормой

$$
\|f\|_{W^{p, 1}(\Omega)}:=\|f\|_{L^{p}(\Omega)}+\|\nabla f\|_{L^{p}(\Omega)}
$$

где $\|\cdot\|_{L^{p}(\Omega)}$ обозначает $L^{p}(\Omega)$-норму скалярных или векторных функций. Замыкание $C_{0}^{\infty}(\Omega)$ в $W^{p, 1}(\Omega)$ обозначается символом $W_{0}^{p, 1}(\Omega)$. Через $W_{\mathrm{loc}}^{p, 1}(\Omega)$ и $L_{\mathrm{loc}}^{p}(\Omega)$ обозначим пространства функций, принадлежащих $W^{p, 1}(B)$ и $L^{p}(B)$ соответственно для каждого шара $B$ с замыканием в $\Omega$.

Если $\mu$ - борелевская мера (возможно, знакопеременная) на области $\Omega$ в $\mathbf{R}^{d}$ или $\mathbf{R}^{d} \times(0,1)$ и $|\mu|$ - ее вариация, то $L^{p}(\mu)$ будет обозначать пространство $L^{p}(|\mu|)$, а $L_{\text {loc }}^{p}(\mu)$ - множество таких функций $f$ на $\Omega$, что $\zeta f \in L^{p}(\mu)$ для всех $\zeta \in C_{0}^{\infty}(\Omega)$.

Пусть $J \subset \mathbf{R}^{1}$ - интервал и $U$ - открытое множество в $\mathbf{R}^{d}$. Через $\mathbf{H}^{p, 1}(U, J)$ обозначим пространство всех измеримых функций $u$ на $U \times J$ таких, что $u(\cdot, t) \in W^{p, 1}(U)$, причем конечна норма

$$
\|u\|_{\mathbf{H}^{p, 1}(U, J)}=\left(\int_{J}\|u(\cdot, t)\|_{W^{p, 1}(U)}^{p} d t\right)^{1 / p}
$$

Пространство $\mathbf{H}_{0}^{p, 1}(U, J)$ определяется аналогично с заменой $W^{p, 1}(U)$ на $W_{0}^{p, 1}(U)$. Сопряженное к $\mathbf{H}_{0}^{p, 1}(U, J)$ пространство обозначается $\mathbf{H}^{p^{\prime},-1}(U, J)$, где $p^{\prime}:=p(p-1)^{-1}$. В связи с параболическими уравнениями полезно ввести также следующие пространства. Пусть $\mathscr{H}^{p, 1}(U, J)$ - пространство всех функций $u \in \mathbf{H}^{p, 1}(U, J)$, для которых $\partial_{t} u \in \mathbf{H}^{p,-1}(U, J)$ и конечна норма

$$
\|u\|_{\mathscr{H}^{p, 1}(U, J)}=\left\|\partial_{t} u\right\|_{\mathbf{H}^{p,-1}(U, J)}+\|u\|_{\mathbf{H}^{p, 1}(U, J)}
$$

Для функций $u$ от $(x, t)$ будем писать

$$
\nabla u:=\nabla_{x} u:=\left(\partial_{x_{1}} u, \ldots, \partial_{x_{d}} u\right)
$$

Через $|\Omega|$ обозначается лебеговский объем множества $\Omega$. Норму вектора $v$ в $\mathbf{R}^{d}$ обозначаем через $|v|$.

Операторное отображение $A$ на открытом множестве называется локально равномерно невырожденным, если отображение $A^{-1}$ локально ограничено. 
2. Существование плотностей. В этом пункте мы приведем модификации некоторых результатов из [1] о существовании непрерывных плотностей. Следуюший результат - теорема 2.8 из работы [1] - был получен для уравнений с дифференциальным оператором в недивергентной форме и с $\beta_{2}=0$. Для удобства читателя укажем необходимые изменения в доказательстве.

Пусть $B_{R}$ - открытый шар радиуса $R$ в $\mathbf{R}^{d}$.

Теорема 2.1. Пусть $p>d, r \in\left(p^{\prime}, \infty\right) u \mu-$ мера на $B_{R}$ c nлотностью $\varrho \in L_{\mathrm{loc}}^{r}\left(B_{R}\right)$. Пусть $a^{i j} \in W_{\mathrm{loc}}^{p, 1}\left(B_{R}\right), \beta_{1} \in L_{\mathrm{loc}}^{p}\left(B_{R}\right), \beta_{2} \in L_{\mathrm{loc}}^{p}(\mu)$, причем $A$ локально равномерно невырождено на $B_{R}$. Предположим также, что для всякого $\varphi \in C_{0}^{\infty}\left(B_{R}\right)$ мы имеем

$$
\left|\int_{B_{R}} a^{i j} \partial_{x_{i}} \partial_{x_{j}} \varphi d \mu\right| \leqslant \int_{B_{R}}\left(|\varphi|+\left|\nabla_{x} \varphi\right|\right)\left(\left|\beta_{1} \varrho\right|+\left|\beta_{2} \varrho\right|\right) d x .
$$

Тогда $\varrho \in W_{\mathrm{loc}}^{p, 1}\left(B_{R}\right)$ и $\varrho$ имеет локально гёльдерову версию.

Д о к а з а т ель с т в о. Поскольку $r>p^{\prime}$, мы имеем $p r>p+r$. Тогда

$$
q=\frac{p r}{p r-p-r}>1 \quad \text { и } \quad q^{\prime}=\frac{p r}{p+r}>1 .
$$

Согласно неравенству Гёльдера имеем $\beta_{1} \varrho \in L_{\text {loc }}^{q^{\prime}}\left(B_{R}\right)$. То же самое верно для $\beta_{2} \mu$, поскольку

$$
\left|\beta_{2}\right|^{q^{\prime}}|\varrho|^{q^{\prime}}=\left|\beta_{2}\right|^{p r /(p+r)}|\varrho|^{r /(p+r)}|\varrho|^{(p r-r) /(p+r)},
$$

где $\left|\beta_{2}\right|^{p r /(p+r)}|\varrho|^{r /(p+r)} \in L_{\text {loc }}^{s}\left(B_{R}\right)$ и $|\varrho|^{(p r-r) /(p+r)} \in L_{\text {loc }}^{s^{\prime}}\left(B_{R}\right)$, причем $s=$ $(p+r) / r$. Значит, $\left|\beta_{1} \varrho\right|+\left|\beta_{2} \varrho\right| \in L_{\text {loc }}^{q^{\prime}}\left(B_{R}\right)$. Теперь то же рассуждение, что и в теореме 2.8 работы [1], завершает доказательство.

Следствие 2.1. Пусть $\mu$ - локально конечная борелевская мера на $B_{R}$. Пусть $A$ локально невырождено в $B_{R}$, причем $a^{i j} \in W_{\mathrm{loc}}^{p, 1}\left(B_{R}\right)$, где $p>d$ u выббраны непрерывные версии $a^{i j}, \partial_{x_{i}} a^{i j} \in L_{\text {loc }}^{p}(\mu), u$ пусть $b^{i}, c \in L_{\mathrm{loc}}^{p}(\mu)$. Предположим, что

$$
\int_{B_{R}}\left[\left(a^{i j} \partial_{x_{i}} \partial_{x_{j}} \varphi+\partial_{x_{i}} a^{i j} \partial_{x_{j}} \varphi+b^{i} \partial_{x_{i}} \varphi+c \varphi\right] d \mu=0 \quad \forall \varphi \in C_{0}^{\infty}\left(B_{R}\right) .\right.
$$

Тогда $\mu$ имеет плотность из $W_{\mathrm{loc}}^{p, 1}\left(B_{R}\right)$, которая локально гёльдерова.

Доказате льст в о. Достаточно взять $\beta_{1}=\left|\partial_{x_{i}} a^{i j}\right|$ и $\beta_{2}=$ $|b|+|c|$ и применить теорему 2.1 .

Мы распространим эти результаты на параболический случай. Наша стратегия будет по сушеству такой же, как и в эллиптическом случае, но из-за некоторых дополнительных технических сложностей мы приведем полные доказательства. 
Пусть $\Omega_{T}=\Omega \times(0, T), T>0$, и пусть $A(\cdot, \cdot)=\left(a^{i j}(\cdot, \cdot)\right)_{i, j=1}^{d}-$ борелевское отображение на $\Omega_{T}$ со значениями в пространстве неотрицательных симметричных операторов в $\mathbf{R}^{d}$. Пусть $\omega_{0} \in \Omega-$ фиксированная точка и $B_{R} \subset \Omega$ - шар радиуса $R$ с центром $\omega_{0}$. Положим $B_{R, T}:=B_{R} \times(0, T)$.

Мы будем предполагать, что функции $a^{i j}(x, t)$ непрерывны по $x$ равномерно по $t$. Заметим, что $a^{i j}$ имеет модификацию с таким свойством при условии, что

$$
\sup _{t}\left\|a^{i j}(\cdot, t)\right\|_{W^{p, 1}\left(B_{R}\right)}<\infty, \quad \text { где } \quad p>d .
$$

Следующий результат - теорема 3.7 из [1].

Теорема 2.2. Пусть $d \geqslant 2, p>d, q \in\left[p^{\prime},+\infty\right)$. Пусть А равномерно ограничено и равномерно невырождено, и пусть выполнено (2.1). Предположим, что $\mu$ - конечная мера на $B_{R, T}$ такая, что при некотором $N>0$ имеем

$$
\left|\int\left[\partial_{t} \varphi+a^{i j} \partial_{x_{i}} \partial_{x_{j}} \varphi\right] d \mu\right| \leqslant N\left\|\nabla_{x} \varphi\right\|_{L^{q}\left(B_{R, T}\right)} \quad \forall \varphi \in C_{0}^{\infty}\left(B_{R, T}\right) .
$$

Тогда $\mu \in \mathbf{H}^{q^{\prime}, 1}\left(B_{R^{\prime}},\left[t_{0}, t_{1}\right]\right) u \mu \in \mathscr{H}^{q^{\prime}, 1}\left(B_{R^{\prime}},\left[t_{0}, t_{1}\right]\right) n p u R^{\prime}<R u\left[t_{0}, t_{1}\right] \subset$ $(0, T)$.

Теперь мы докажем аналог теоремы 3.8 работы [1], отличающийся тем, что условие интегрируемости коэффициента $\beta_{2}$ (отсутствовавшего в цитированной теореме) выражено в терминах меры $\mu$, а не меры Лебега.

Теорема 2.3. Пусть $A$ - локально ограниченное и локально равномерно невырожденное отображение на $B_{R, T}$, u пусть выполнено (2.1), где мь предполагаем теперь, что $p>d+2$. Пусть $\mu-$ конечная борелевская мера на $B_{R, T}$ с плотностью $\varrho \in L^{r}\left(B_{R, T}\right)$ при некотором $r>p^{\prime}$. Пусть $\beta_{1} \in L_{\mathrm{loc}}^{p}\left(B_{R, T}\right) u \beta_{2} \in L_{\mathrm{loc}}^{p}(\mu)$. Предположим, ито для всех $\varphi \in C_{0}^{\infty}\left(B_{R, T}\right)$ мьи имеем

$$
\left|\int_{B_{R, T}}\left[\partial_{t} \varphi+a^{i j} \partial_{x_{i}} \partial_{x_{j}} \varphi\right] d \mu\right| \leqslant \int_{B_{R, T}}\left(|\varphi|+\left|\nabla_{x} \varphi\right|\right)\left(\left|\beta_{1} \varrho\right|+\left|\beta_{2} \varrho\right|\right) d x d t .
$$

Тогда $\varrho$ имеет версию, которая локально гёльдерова на $B_{R} \times(0, T)$ и принадлежит классам $\mathbf{H}^{p, 1}\left(B_{R^{\prime}},\left[T_{0}, T_{1}\right]\right)$ u $\mathscr{H}^{p, 1}\left(B_{R^{\prime}},\left[T_{0}, T_{1}\right]\right)$ для всякого $R^{\prime}<R$ и всякого отрезка $\left[T_{0}, T_{1}\right] \subset(0, T)$.

Д ок аз а те л с т в о. Мы модифицируем доказательство теоремы 3.8 из [1]. Зафиксируем число $R^{\prime}<R$ и отрезок $\left[T_{0}, T_{1}\right] \subset(0, T)$. Из неравенства Гёльдера с показателями $p / \theta$ и $p /(p-\theta)$, применяемого к произведению

$$
\left|\beta_{2}\right|^{\theta}|\varrho|^{\theta}=\left|\beta_{2}\right|^{\theta}|\varrho|^{\theta / p}|\varrho|^{\theta-\theta / p}
$$


вытекает, что $\beta_{2} \varrho \in L_{\mathrm{loc}}^{\theta}\left(B_{R, T}\right)$, если $(p \theta-\theta)(p-\theta)^{-1}=r$, т.е. $\theta=p r(p+$ $r-1)^{-1}$. Положим $s:=p r(p+r)^{-1}$. Тогда $\theta>s$ и потому $\beta_{2} \varrho \in L_{\mathrm{loc}}^{s}\left(B_{R, T}\right)$. Кроме того, $\left|\beta_{1} \varrho\right| \in L_{\text {loc }}^{s}\left(B_{R, T}\right)$ также в силу неравенства Гёльдера и условий на $\beta_{1}$ и $\varrho$. При этом $q:=s^{\prime}>p^{\prime}$. Значит, (2.2) выполнено при $q=$ $s^{\prime}>p^{\prime}$. Это вытекает из неравенства Гёльдера с показателями $q$ и $q^{\prime}=s$ с учетом того факта, что для $\varphi \in C_{0}^{\infty}\left(B_{R, T}\right)$ интеграл от $(|\varphi|+|\nabla \varphi|)^{q}$ по $B_{R, T}$ оценивается через $C\left\|\nabla_{x} \varphi\right\|_{L^{q}\left(B_{R, T}\right)}^{q}$ с некоторым числом $C$, не зависящим от $\varphi$.

По теореме 2.2 получаем включения $\varrho \in \mathbf{H}^{s, 1}\left(B_{R_{1}},\left[t_{0}, t_{1}\right]\right)$ и $\varrho \in$ $\mathscr{H}^{s, 1}\left(B_{R_{1}},\left[t_{0}, t_{1}\right]\right)$ для всякого отрезка $\left[t_{0}, t_{1}\right] \subset(0, T)$ и всякого $R_{1}<R$. В частности, можно взять любые $t_{0} \in\left(0, T_{0}\right), t_{1} \in\left(T_{1}, T\right), R_{1} \in\left(R^{\prime}, R\right)$.

Напомним теорему вложения для пространств $\mathscr{H}^{p, 1}\left(\mathbf{R}^{d},[0, T]\right)$. Доказательство можно найти, например, в $[12$, следствие 7.6$]$ или $[13$, теорема 7.2] (где ограничение $p>2$ нужно только в случае стохастических соболевских пространств). Пусть $q>p>1$ и $(d+2)(1 / p-$ $1 / q)<1$. Тогда сушествует число $N(d, p, q, T)$ такое, что для всякого $u \in \mathscr{H}^{p, 1}\left(\mathbf{R}^{d},[0, T]\right)$ справедливо неравенство

$$
\|u\|_{L^{q}\left(\mathbf{R}^{d} \times[0, T]\right)} \leqslant N(d, p, q, T)\|u\|_{\mathscr{H}^{p, 1}\left(\mathbf{R}^{d},[0, T]\right)} .
$$

Нетрудно проверить, что то же самое верно для шара вместо $\mathbf{R}^{d}$. Cогласно (2.3), мы получаем, что $\varrho \in L^{r_{1}}\left(B_{R_{1}} \times\left[t_{0}, t_{1}\right]\right)$ для всякого $r_{1}$ такого, что

$$
\frac{1}{r_{1}}>\frac{1}{s}-\frac{1}{d+2} .
$$

Это эквивалентно соотношению

$$
\frac{1}{r_{1}}>\frac{p+r}{p r}-\frac{1}{d+2}=\frac{(d+2)(p+r)-p r}{p r(d+2)},
$$

которое может быть записано как

$$
r_{1}<r \frac{p(d+2)}{(d+2)(p+r)-p r},
$$

при условии, что $(d+2)(p+r)-p r>0$; если $(d+2)(p+r)-p r \leqslant 0$, то в качестве $r_{1}$ можно взять любое число, большее единицы.

Можно выбрать $r_{1}$ достаточно близким к $r p(d+2)((d+2)(p+r)-p r)^{-1}$ таким образом, что

$$
\begin{aligned}
\frac{r_{1}}{r} & =\frac{p(d+2)}{(d+2)(p+r)-p r}=\frac{p}{p+r-p r /(d+2)} \\
& =\frac{p}{p-r(p /(d+2)-1)} \geqslant \frac{p}{p-p /(d+2)+1}>1 .
\end{aligned}
$$


Следовательно, повторяя описанную выше процедуру конечное число раз, мы придем к ситуации с $(d+2)(p+r)-p r \leqslant 0$, когда

$$
\begin{array}{ccc}
\varrho \in L^{r}\left(B_{R_{0}} \times\left[\tau_{0}, \tau_{1}\right]\right), \quad \text { для всех } & r \in(1,+\infty), \\
\text { где } 0<\tau_{0}<T_{0}, \quad T_{1}<\tau_{1}<T, & R^{\prime}<R_{0}<R .
\end{array}
$$

Значит, $\varrho \in \mathbf{H}^{s, 1}\left(B_{R_{0}},\left[\tau_{0}, \tau_{1}\right]\right)$ и $\varrho \in \mathscr{H}^{s, 1}\left(B_{R_{0}},\left[\tau_{0}, \tau_{1}\right]\right)$ для каждого $s<p$. Выберем $s>d+2, \alpha>0$ и $\kappa>0$ так, что $\kappa>\alpha>1 / s$ и $s(1-2 \kappa)>d$, что возможно, поскольку $p>d+2$. Тогда, как вытекает из [12, теорема 7.2$]$ и теоремы вложения Соболева, при некотором $\gamma>0$ функция $\varrho$ имеет версию из класса $C^{\alpha-1 / s}\left(\left[\tau_{0}, \tau_{1}\right], C^{\gamma}\left(B_{R_{0}}\right)\right)$, состоящего из гёльдеровых порядка $\alpha-1 / s$ отображений отрезка $\left[\tau_{0}, \tau_{1}\right]$ в пространство $C^{\gamma}\left(B_{R_{0}}\right)$ функций на $B_{R_{0}}$, удовлетворяющих условию Гёльдера порядка $\gamma$. Итак, функция $\varrho$ имеет гёльдерову версию.

Следствие 2.2. Пусть $p>d+2$, и пусть $A$ локально равномерно ограничено и локально равномерно невырождено на $\Omega_{T}$, причем выполнено (2.1) с некоторым числом $p>d+2$ для каждого шара $B_{R}$ с компактным замыканием в $\Omega$. Предположим, что $\mu$-локально конечная знакопеременная борелевская мера на $\Omega_{T}$ такая, что $b^{i}, c \in L_{\mathrm{loc}}^{p}(\mu) u$

$\int_{\Omega_{T}}\left[\partial_{t} \varphi+a^{i j} \partial_{x_{i}} \partial_{x_{j}} \varphi+\partial_{x_{i}} a^{i j} \partial_{x_{j}} \varphi+b^{i} \partial_{x_{i}} \varphi+c \varphi\right] d \mu=0 \quad \forall \varphi \in C_{0}^{\infty}\left(\Omega_{T}\right)$.

Тогда $\mu$ имеет локально гёльдерову плотность, принадлежащую пространствам $\mathbf{H}^{p, 1}(U, J)$ и $\mathscr{H}^{p, 1}(U, J)$ для всякого интервала $J$ и всякого открытого множества $U$, для которых $U \times J$ имеет компактное замькание в $\Omega_{T}$.

Д о к а з а т ел ь с т в о. Согласно [1, следствие 3.2], мера $\mu$ имеет плотность из $L_{\text {loc }}^{r}\left(\Omega_{T}\right)$ при всяком $r<(d+2)^{\prime}$, в частности, при некотором $r>p^{\prime}$. Значит, применима теорема 2.3 с $\beta_{1}=\left|\partial_{x_{i}} a^{i j}\right|$ и $\beta_{2}=|b|+|c|$.

3. Строгая положительность плотностей. Предыдущие результаты дают существование и соболевскую регулярность плотностей решений слабых эллиптических и параболических уравнений. Следующие теоремы дают достаточные условия строгой положительности плотностей решений. Далее мы рассматриваем только неотрицательные меры. Для начала напомним похожий результат в эллиптическом случае. Он был получен в [2].

Теорема 3.1. Пусть $\mu$ - ненулевая локально конечная неотрииательная борелевская мера на $\mathbf{R}^{d}$. Пусть $A$ - локально равномерно невырожденное отображение с $a^{i j} \in W_{\text {loc }}^{p, 1}\left(\mathbf{R}^{d}\right)$, где $p>d$, u nусть для каждого компакта $K$ существует иисло $\kappa=\kappa(K)>0$ maкое, что Функиия $\exp (\kappa|b|)$ интегрируема относительно н на $K$. Предположим, что $\Theta \in L_{\text {loc }}^{p}(\mu)$ и что существует такая измеримая функиия $\Psi \geqslant 0$, 
что $\Theta \geqslant-\Psi$ u $\exp (\Psi) \in L_{\text {loc }}^{1}(\mu)$. Предположим также, что $\mu$ удовлетворяет уравнению $\mathscr{L}^{*} \mu=0$ на $\mathbf{R}^{d}$, где

$$
\mathscr{L} u(x):=\partial_{x_{i}}\left(a^{i j}(x) \partial_{x_{j}} u(x)\right)+b^{i}(x) \partial_{x_{i}} u(x)+\Theta(x) u(x) .
$$

Тогда мера $\mu$ имеет непрерывную строго положительную плотность $\varrho$.

Заметим, что существование непрерывной плотности немедленно вытекает из следствия 2.1 .

З а м е ч а н и е 3.1. Пусть известно, что ненулевая неотрицательная мера $\mu$ имеет локально ограниченную плотность $\varrho \in W_{\mathrm{loc}}^{2,1}\left(\mathbf{R}^{d}\right)$. Вместо уравнения (1.1) можно рассматривать неравенство $\mathscr{L}^{*} \mu \leqslant 0$, где

$$
\mathscr{L} u(x):=\partial_{x_{i}}\left(a^{i j}(x) \partial_{x_{j}} u(x)\right)+b^{i}(x) \partial_{x_{i}} u(x)-\Psi(x) u(x) .
$$

Неравенство понимается следующим образом:

$$
\int_{\mathbf{R}^{d}} \mathscr{L} \varphi(x) d \mu \leqslant 0
$$

для всякой функции $\varphi \in C_{0}^{\infty}\left(\mathbf{R}^{d}\right)$ такой, что $\varphi \geqslant 0$. Предположим, что $A$ локально равномерно невырождено, причем $a^{i j} \in W_{\mathrm{loc}}^{p, 1}\left(\mathbf{R}^{d}\right)$, где $p>d$, $\Psi \geqslant 0$ и $\exp (\Psi) \in L_{\text {loc }}^{1}(\mu)$. Пусть $b$ удовлетворяет тому же условию локальной экспоненциальной интегрируемости, что и в теореме. Тогда $\varrho$ имеет версию, локально отделенную от нуля.

Рассмотрим параболический случай. Пусть $\mu-$ неотрицательная локально конечная борелевская мера на $\mathbf{R}^{d} \times(0,1)$, удовлетворяющая уравнению (1.1).

Будем предполагать, что отображение $A(x, t)=\left(a^{i j}(x, t)\right)_{1 \leqslant i, j \leqslant d}$ со значениями в пространстве положительных симметричных матриц и векторное поле $b(x, t)=\left(b^{i}(x, t)\right)_{1 \leqslant i \leqslant d}$ удовлетворяют следующим условиям:

(C1) при некотором $p>d+2$ функции $a^{i j}$ входят в $\mathbf{H}^{p, 1}(U, J)$ для всякого шара $U$ и всякого интервала $J$ с компактным замыканием в $(0,1)$;

(C2) для всякого компакта $K \subset \mathbf{R}^{d} \times(0,1)$ существуют числа $m(K), M(K)>0$ такие, что для всех $(x, t) \in K$ и всех $y \in \mathbf{R}^{d}$ имеем

$$
m(K)|y|^{2} \leqslant \sum_{1 \leqslant i, j \leqslant d} a^{i j}(x, t) y_{i} y_{j} \leqslant M(K)|y|^{2} ;
$$

(C3) для всякого компакта $K \subset \mathbf{R}^{d} \times(0,1)$ существует такое число $\kappa(K)>0$, что функция $\exp \left(\kappa(K)|b|^{2}\right)$ интегрируема относительно меры $\mu$ на $K$.

Пусть $K(r)=K(x, r)$ обозначает куб с длиной ребра $r$ и центром $x, \mathrm{y}$ которого ребра параллельны координатным осям. Сначала мы получим априорные оценки. 
При $q \in(-\infty, 1]$ и $s>0$ положим

$$
h_{q}(s):=-\frac{1}{s|\ln s|^{2 q-1}} .
$$

Тогда $h_{q}(s)=H_{q}^{\prime}(s)$, где $H_{q}(s):=(2-2 q)^{-1}|\ln s|^{2-2 q}$ при $q<1$ и $H_{1}(s):=$ $\ln |\ln s|$.

Лемма 3.1. Для всякого $\tau \in\left(0, e^{-2}\right)$ имеем

(i) $h_{q}^{2}(\tau) / h_{q}^{\prime}(\tau) \leqslant|\ln \tau|^{2-2 q}$,

(ii) $\tau^{2} h_{q}^{\prime}(\tau)\left|\ln h_{q}^{\prime}(\tau)\right| \leqslant 9(1+|q|)^{2}|\ln \tau|^{2-2 q}$.

Доказат ельст в о. В силу равенства $h_{q}^{\prime}(s)=(|\ln s|+1-$ 2q) $s^{-2}|\ln s|^{-2 q}$ мы имеем

$$
\frac{h_{q}^{2}(\tau)}{h_{q}^{\prime}(\tau)}=\frac{\tau^{2}|\ln \tau|^{2 q}}{\tau^{2}|\ln \tau|^{4 q-2}(|\ln \tau|-2 q+1)}=\frac{|\ln \tau|^{2-2 q}}{|\ln \tau|-2 q+1} .
$$

Заметим, что если $\tau \in\left(0, e^{-2}\right)$ и $q \leqslant 1$, то $|\ln \tau|-2 q+1 \geqslant 1$. Это дает (i). Проверим неравенство (ii). Мы имеем

$$
\begin{array}{r}
\tau^{2} h_{q}^{\prime}(\tau)\left|\ln h_{q}^{\prime}(\tau)\right| \leqslant \tau^{2}(|\ln \tau|-2 q+1)(2|\ln \tau|+2|q| \ln |\ln \tau| \\
+\ln || \ln \tau|+2 q-1|)\left(\tau^{2}|\ln \tau|^{2 q}\right)^{-1}
\end{array}
$$

Поэтому получаем $\tau^{2} h_{q}^{\prime}(\tau)\left|\ln h_{q}^{\prime}(\tau)\right| \leqslant 9(1+|q|)^{2}|\ln \tau|^{2-2 q}$, что завершает доказательство.

Пусть $\Omega$ - произвольная область в $\mathbf{R}^{d}$, и пусть $\left[T_{0}, T_{1}\right] \subset(0,1)$. Согласно следствию 2.2 , мера $\mu$ имеет плотность $\varrho \in L^{\infty}\left(\Omega \times\left[T_{0}, T_{1}\right]\right)$. Умножив $\varrho$ на $\left(2\|\varrho\|_{L^{\infty}}\right)^{-1} e^{-2}$, будем считать, что

$$
\|\varrho\|_{L^{\infty}} \leqslant 2^{-1} e^{-2} \text {. }
$$

Положим $\varrho_{k}:=\varrho+k^{-1}$, где $k>2 e^{2}$. Заметим, что $k^{-1} \leqslant \varrho_{k} \leqslant e^{-2}$.

Лемма 3.2. Пусть $\eta \in C_{0}^{1}\left(\Omega \times\left[T_{0}, T_{1}\right]\right), T_{0}<\tau_{1}<\tau_{2}<T_{1} u$ $0<\gamma<\kappa$, где $\kappa=\kappa\left(\Omega \times\left[T_{0}, T_{1}\right]\right)$ - число из условия (C3). Тогда выполнены следуюшие оченки.

Eсли $q=1$ и $\eta(t, x)=\eta_{0}(x)$ nрu $t \in\left[\tau_{1}, \tau_{2}\right]$, mo

$$
\begin{gathered}
\int_{\Omega}\left[\ln \left(\left|\ln \varrho_{k}\left(x, \tau_{2}\right)\right|\right) \eta_{0}^{2}(x)-\ln \left(\left|\ln \varrho_{k}\left(x, \tau_{1}\right)\right|\right) \eta_{0}^{2}(x)\right] d x \\
\quad+\frac{m}{3} \int_{\tau_{1}}^{\tau_{2}} \int_{\Omega}|\nabla \ln | \ln \varrho_{k}||^{2} \eta_{0}^{2} d x d t \\
\leqslant C\left[\left(1+\gamma^{-1}\right) \int_{\tau_{1}}^{\tau_{2}} \int_{\Omega}\left(\eta_{0}^{2}+\left|\nabla \eta_{0}\right|^{2}\right) d x d t\right. \\
\left.\quad+\gamma^{-1}\|\varrho\|_{L^{\infty}} \int_{\tau_{1}}^{\tau_{2}} \int_{\Omega} e^{\gamma|b|^{2}} \varrho \eta_{0}^{2} d x d t\right] .
\end{gathered}
$$


Ecлu $q<1$, mo

$$
\begin{aligned}
& \frac{1}{2-2 q} \int_{\Omega}\left[\left|\ln \varrho_{k}\left(x, \tau_{2}\right)\right|^{2-2 q} \eta^{2}\left(x, \tau_{2}\right)-\left|\ln \varrho_{k}\left(x, \tau_{1}\right)\right|^{2-2 q} \eta^{2}\left(x, \tau_{1}\right)\right] d x \\
& +\left.\left.\frac{m}{3(1-q)^{2}} \int_{\tau_{1}}^{\tau_{2}} \int_{\Omega}|\nabla| \ln \varrho_{k}\right|^{1-q}\right|^{2} \eta^{2} d x d t \\
& \leqslant 2 \int_{\tau_{1}}^{\tau_{2}} \int_{\Omega}|\eta|\left|\eta_{t}\right|\left|\ln \varrho_{k}\right|^{2-2 q} d x d t \\
& +C\left[\left(1+\gamma^{-1}\right)(1+|q|)^{2} \int_{\tau_{1}}^{\tau_{2}} \int_{\Omega}\left(\eta^{2}+|\nabla \eta|^{2}\right)\left|\ln \varrho_{k}\right|^{2-2 q} d x d t\right. \\
& \left.\quad+\gamma^{-1}\|\varrho\|_{L^{\infty}} \int_{\tau_{1}}^{\tau_{2}} \int_{\Omega} e^{\gamma|b|^{2}} \varrho \eta^{2} d x d t\right] .
\end{aligned}
$$

$B$ этих оченках $m=m\left(\Omega \times\left[T_{0}, T_{1}\right]\right)$ - число из условия $(\mathrm{C} 2)$ и $C$ число, зависящее только от $d, m\left(\Omega \times\left[T_{0}, T_{1}\right]\right)$ u $M\left(\Omega \times\left[T_{0}, T_{1}\right]\right)$.

Д ока за т е л ь с т в о. Для каждой функции $\varphi \in \mathbf{H}_{0}^{2,1}(\Omega,(0,1))$ имеет место следующее тождество:

$$
\int_{0}^{1} \int_{\Omega}\left[-\partial_{t} \varphi \varrho+(A \nabla \varrho, \nabla \varphi)\right] d x d t=\int_{0}^{1} \int_{B}(b, \nabla \varphi) \varrho d x d t
$$

Пусть $\omega_{r}(s):=r^{-1} \omega\left(s r^{-1}\right)$, где $0 \leqslant \omega \leqslant 1, \omega \in C_{0}^{1}\left(\mathbf{R}^{1}\right), \operatorname{supp} \omega \subset\left[\frac{1}{2}, \frac{3}{4}\right]$ и

$$
\int_{0}^{1} \omega(s) d s=1
$$

Положим также

$$
\varrho_{r}(x, t):=\int_{0}^{1} \omega_{r}(t-s) \varrho(x, s) d s, \quad t>r .
$$

Тогда функция $\varrho_{r}$ непрерывно дифференцируема по $t$. Согласно (3.3), функция $\varrho_{r}$ удовлетворяет интегральному равенству

$$
\int_{0}^{1} \int_{\Omega}\left[\psi \partial_{t} \varrho_{r}+\left((A \nabla \varrho) * \omega_{r}, \nabla \psi\right)\right] d x d t=\int_{0}^{1} \int_{\Omega}\left((b \varrho) * \omega_{r}, \nabla \psi\right) d x d t
$$

для каждой функции $\psi \in \mathbf{H}_{0}^{2,1}(\Omega,(0,1))$ такой, что $\psi(x, t)=0$ при $t \in(0, r]$. Заметим, что свертка $(b \varrho) * \omega_{r}$ корректно определена, поскольку по теореме Фубини $b(x, \cdot) \varrho(x, \cdot) \in L^{1}[\delta, 1-\delta]$ при почти всех $x$ и всех $\delta>0$.

Пусть $k>0$ фиксировано и $\alpha_{0}>0$ выбрано так, что $\left[T_{0}, T_{1}\right] \subset$ $\left[\alpha_{0}, 1-\alpha_{0}\right]$ и $\eta(x, t)=0$ при $t \notin\left[\alpha_{0}, 1-\alpha_{0}\right]$. Далее рассматриваем $r<\alpha_{0}$. Положим

$$
\varrho_{r, k}:=\varrho_{r}+k^{-1}, \quad \psi:=I_{\left[\tau_{1}, \tau_{2}\right]} h_{q}\left(\varrho_{r, k}\right) \eta^{2}
$$


где $I_{\left[\tau_{1}, \tau_{2}\right]}$ обозначает индикатор отрезка $\left[\tau_{1}, \tau_{2}\right]$. Подставляя такую функцию $\psi$ в (3.4) и используя формулу Ньютона-Лейбница, находим

$$
\begin{aligned}
\int_{\Omega} & {\left[H_{q}\left(\varrho_{r, k}\left(x, \tau_{2}\right)\right) \eta^{2}\left(x, \tau_{2}\right)-H_{q}\left(\varrho_{r, k}\left(x, \tau_{1}\right)\right) \eta^{2}\left(x, \tau_{1}\right)\right] d x } \\
& +\int_{\tau_{1}}^{\tau_{2}} \int_{\Omega}\left((A \nabla \varrho) * \omega_{r}, \nabla \varrho_{r, k}\right) h_{q}^{\prime}\left(\varrho_{r, k}\right) \eta^{2} d x d t \\
= & I(r, k)+J(r, k)+L(r, k)+N(r, k),
\end{aligned}
$$

где

$$
\begin{aligned}
I(r, k) & =-2 \int_{\tau_{1}}^{\tau_{2}} \int_{\Omega}\left((A \nabla \varrho) * \omega_{r}, \nabla \eta\right) h_{q}\left(\varrho_{r, k}\right) \eta d x d t, \\
J(r, k) & =\int_{\tau_{1}}^{\tau_{2}} \int_{\Omega}\left((b \varrho) * \omega_{r}, \nabla \varrho_{r, k}\right) h_{q}^{\prime}\left(\varrho_{r, k}\right) \eta^{2} d x d t, \\
L(r, k) & =2 \int_{\tau_{1}}^{\tau_{2}} \int_{\Omega}\left((b \varrho) * \omega_{r}, \nabla \eta\right) h_{q}\left(\varrho_{r, k}\right) \eta d x d t, \\
N(r, k) & =2 \int_{\tau_{1}}^{\tau_{2}} \int_{\Omega} H_{q}\left(\varrho_{r, k}\right) \eta \partial_{t} \eta d x d t .
\end{aligned}
$$

Функция $\left|H\left(\varrho_{r, k}\right)\right|$ на носителе функции $\eta$ оценивается некоторым числом равномерно по всем $r \leqslant \alpha_{0}$. Следовательно, согласно теореме Лебега о мажорируемой сходимости, при $r \rightarrow 0$ мы имеем

$$
\begin{aligned}
\int_{\Omega} H_{q}\left(\varrho_{r, k}\left(x, \tau_{i}\right)\right) \eta^{2}\left(x, \tau_{i}\right) d x & \longrightarrow \int_{\Omega} H_{q}\left(\varrho_{k}\left(x, \tau_{i}\right)\right) \eta^{2}\left(x, \tau_{i}\right) d x, \quad i=1,2, \\
\int_{\tau_{1}}^{\tau_{2}} \int_{\Omega} H_{q}\left(\varrho_{r, k}\right) \eta \partial_{t} \eta d x d t & \longrightarrow \int_{\tau_{1}}^{\tau_{2}} \int_{\Omega} H_{q}\left(\varrho_{k}\right) \eta \partial_{t} \eta d x d t .
\end{aligned}
$$

Заметим, что $\left|h_{q}^{\prime}\left(\varrho_{r, k}\right)\right| \leqslant C(k)$ для некоторого числа $C(k)$ и всех $r \in(0,1)$. Функции $A \nabla \varrho, b \varrho, \nabla \varrho_{r, k}$ входят в $L^{2}\left(\Omega \times\left[T_{0}, T_{1}\right]\right)$ при $r<\alpha_{0}$. Поэтому при $r \rightarrow 0$ мы получаем

$$
\begin{gathered}
\left\|(A \nabla \varrho) * \omega_{r}-A \nabla \varrho\right\|_{L^{2}\left(\Omega \times\left[T_{0}, T_{1}\right]\right)} \longrightarrow 0, \quad\left\|(b \varrho) * \omega_{r}-b \varrho\right\|_{L^{2}\left(\Omega \times\left[T_{0}, T_{1}\right]\right)} \longrightarrow 0 \\
\left\|\nabla \varrho_{r, k}-\nabla \varrho_{k}\right\|_{L^{2}\left(\Omega \times\left[T_{0}, T_{1}\right]\right)} \longrightarrow 0 .
\end{gathered}
$$

Члены

$$
\int_{\tau_{1}}^{\tau_{2}} \int_{\Omega}\left((A \nabla \varrho) * \omega_{r}, \nabla \varrho_{r, k}\right) h_{q}^{\prime}\left(\varrho_{r, k}\right) \eta^{2} d x d t
$$

и $I(r, k), J(r, k), L(r, k)$ сходятся при $r \rightarrow 0$. Таким образом, имеем

$$
\begin{aligned}
\int_{\Omega} & {\left[H_{q}\left(\varrho_{k}\left(x, \tau_{2}\right)\right) \eta^{2}\left(x, \tau_{2}\right)-H_{q}\left(\varrho_{k}\left(x, \tau_{1}\right)\right) \eta^{2}\left(x, \tau_{1}\right)\right] d x } \\
& \quad+\int_{\tau_{1}}^{\tau_{2}} \int_{\Omega}(A \nabla \varrho, \nabla \varrho) h_{q}^{\prime}\left(\varrho_{k}\right) \eta^{2} d x d t=I(k)+J(k)+L(k)+N(k),
\end{aligned}
$$


где

$$
\begin{aligned}
I(k) & =-2 \int_{\tau_{1}}^{\tau_{2}} \int_{\Omega}(A \nabla \varrho, \nabla \eta) h_{q}\left(\varrho_{k}\right) \eta d x d t \\
J(k) & =\int_{\tau_{1}}^{\tau_{2}} \int_{\Omega}(b \varrho, \nabla \varrho) h_{q}^{\prime}\left(\varrho_{k}\right) \eta^{2} d x d t \\
L(k) & =2 \int_{\tau_{1}}^{\tau_{2}} \int_{\Omega}(b \varrho, \nabla \eta) h_{q}\left(\varrho_{k}\right) \eta d x d t \\
N(k) & =2 \int_{\tau_{1}}^{\tau_{2}} \int_{\Omega} H_{q}\left(\varrho_{k}\right) \eta \eta_{t} d x d t .
\end{aligned}
$$

Оценим каждый член в правой части (3.5) отдельно. Пусть $\varepsilon>0$. Тогда

$$
I(k) \leqslant \varepsilon \int_{\tau_{1}}^{\tau_{2}} \int_{\Omega}|\nabla \varrho|^{2} h_{q}^{\prime}\left(\varrho_{k}\right) \eta^{2} d x d t+\varepsilon^{-1} M^{2} \int_{\tau_{1}}^{\tau_{2}} \int_{\Omega}|\nabla \eta|^{2} \frac{h_{q}^{2}\left(\varrho_{k}\right)}{h_{q}^{\prime}\left(\varrho_{k}\right)} d x d t .
$$

Согласно первому утверждению леммы 3.1, мы имеем

$$
\frac{h_{q}^{2}\left(\varrho_{k}\right)}{h_{q}^{\prime}\left(\varrho_{k}\right)} \leqslant\left|\ln \varrho_{k}\right|^{2-2 q}
$$

Значит,

$$
I(k) \leqslant \varepsilon \int_{\tau_{1}}^{\tau_{2}} \int_{\Omega}|\nabla \varrho|^{2} h_{q}^{\prime}(\varrho) \eta^{2} d x d t+\varepsilon^{-1} M^{2} \int_{\tau_{1}}^{\tau_{2}} \int_{\Omega}|\nabla \eta|^{2}\left|\ln \varrho_{k}\right|^{2-2 q} d x .
$$

Оценим $J(k)$. Имеем

$$
J(k) \leqslant \int_{\tau_{1}}^{\tau_{2}} \int_{\Omega}|\nabla \eta|^{2} \frac{h_{q}^{2}\left(\varrho_{k}\right)}{h_{q}^{\prime}\left(\varrho_{k}\right)} d x d t+\int_{\tau_{1}}^{\tau_{2}} \int_{\Omega}|b|^{2} \varrho^{2} h_{q}^{\prime}\left(\varrho_{k}\right) \eta^{2} d x d t .
$$

Первое слагаемое справа оценивается, как и выше. Рассмотрим второе слагаемое. Заметим, что для всех $\alpha>0, \beta>0, \gamma>0$ выполнено неравенство

$$
\alpha \beta \leqslant \gamma^{-1}\left(e^{\gamma \beta}+\alpha \ln \alpha\right) .
$$

Применяя это неравенство с $\alpha=h_{q}^{\prime}\left(\varrho_{k}\right)$ и $\beta=|b|^{2}$ и учитывая, что $\varrho<\varrho_{k}$, мы получаем

$$
|b|^{2} \varrho^{2} h_{q}^{\prime}\left(\varrho_{k}\right) \leqslant \gamma^{-1} e^{\gamma|b|^{2}} \varrho^{2}+\gamma^{-1} \varrho_{k}^{2} h_{q}^{\prime}\left(\varrho_{k}\right)\left|\ln h^{\prime}\left(\varrho_{k}\right)\right| .
$$

Согласно второму утверждению леммы 3.1, имеем

$$
\varrho_{k}^{2} h_{q}^{\prime}\left(\varrho_{k}\right)\left|\ln h_{q}^{\prime}\left(\varrho_{k}\right)\right| \leqslant 9(1+|q|)^{2}\left|\ln \varrho_{k}\right|^{2-2 q} .
$$

Следовательно,

$$
\begin{aligned}
\int_{\tau_{1}}^{\tau_{2}} \int_{\Omega}|b|^{2} \varrho^{2} h_{q}^{\prime}\left(\varrho_{k}\right) \eta^{2} d x d t \leqslant & \gamma^{-1}\|\varrho\|_{L^{\infty}} \int_{\tau_{1}}^{\tau_{2}} \int_{\Omega} e^{\gamma|b|^{2}} \varrho \eta^{2} d x d t \\
& +9 \gamma^{-1}(1+|q|)^{2} \int_{\tau_{1}}^{\tau_{2}} \int_{\Omega}\left|\ln \varrho_{k}\right|^{2-2 q} \eta^{2} d x d t
\end{aligned}
$$


Итак,

$$
\begin{aligned}
J(k) \leqslant & 9\left(1+\gamma^{-1}\right)(1+|q|)^{2} \int_{\tau_{1}}^{\tau_{2}} \int_{\Omega}\left(\eta^{2}+|\nabla \eta|^{2}\right)\left|\ln \varrho_{k}\right|^{2-2 q} d x d t \\
& +\gamma^{-1}\|\varrho\|_{L^{\infty}} \int_{\tau_{1}}^{\tau_{2}} \int_{\Omega} e^{\gamma|b|^{2}} \varrho \eta^{2} d x d t
\end{aligned}
$$

Оценим $L(k)$. Имеем

$$
L(k) \leqslant \varepsilon \int_{\tau_{1}}^{\tau_{2}} \int_{\Omega}|\nabla \varrho|^{2} h_{q}^{\prime}\left(\varrho_{k}\right) \eta^{2} d x d t+4 \varepsilon^{-1} \int_{\Omega}|b|^{2} \varrho^{2} h_{q}^{\prime}\left(\varrho_{k}\right) \eta^{2} d x d t .
$$

Оценив второе слагаемое в правой части таким же образом, как и выше, мы получаем

$$
\begin{aligned}
L(k) \leqslant & \varepsilon \int_{\tau_{1}}^{\tau_{2}} \int_{\Omega}|\nabla \varrho|^{2} h_{q}^{\prime}\left(\varrho_{k}\right) \eta^{2} d x d t+4 \varepsilon^{-1} \gamma^{-1}\|\varrho\|_{L^{\infty}} \int_{\tau_{1}}^{\tau_{2}} \int_{\Omega} e^{\gamma|b|} \varrho \eta^{2} d x d t \\
& +36 \varepsilon^{-1} \gamma^{-1}(1+|q|)^{2} \int_{\tau_{1}}^{\tau_{2}} \int_{\Omega}\left|\ln \varrho_{k}\right|^{2-2 q} \eta^{2} d x d t .
\end{aligned}
$$

Заметим, что

$$
\int_{\tau_{1}}^{\tau_{2}} \int_{\Omega}(A \nabla \varrho, \nabla \varrho) h_{q}^{\prime}\left(\varrho_{k}\right) \eta^{2} d x d t \geqslant m \int_{\tau_{1}}^{\tau_{2}} \int_{\Omega}|\nabla \varrho|^{2} h_{q}^{\prime}\left(\varrho_{k}\right) \eta^{2} d x d t .
$$

Так как $h_{q}^{\prime}\left(\varrho_{k}\right)=\left(\left|\ln \varrho_{k}\right|-2 q+1\right)\left(\varrho_{k}^{2}\left|\ln \varrho_{k}\right|^{2 q}\right)^{-1}, 0<\varrho_{k}<e^{-2}$ и $q \leqslant 1$, то выполнено неравенство

$$
h_{q}^{\prime}\left(\varrho_{k}\right) \geqslant \frac{1}{\varrho_{k}^{2}\left|\ln \varrho_{k}\right|^{2 q}}
$$

Значит,

$$
\int_{\tau_{1}}^{\tau_{2}} \int_{\Omega}(A \nabla \varrho, \nabla \varrho) h_{q}^{\prime}\left(\varrho_{k}\right) \eta^{2} d x d t \geqslant m \int_{\tau_{1}}^{\tau_{2}} \int_{\Omega} \frac{|\nabla \varrho|^{2}}{\varrho_{k}^{2}\left|\ln \varrho_{k}\right|^{2 q}} \eta^{2} d x d t .
$$

Суммируя полученные выше оценки и полагая $\varepsilon:=m / 3$, приходим $\mathrm{k}$ неравенству

$$
\begin{aligned}
& \left.\int_{\Omega}\left(H_{q}\left(\varrho_{k}\right) \eta^{2}\right)\right|_{\tau_{1}} ^{\tau_{2}} d x+\frac{m}{3} \int_{\tau_{1}}^{\tau_{2}} \int_{\Omega} \frac{|\nabla \varrho|^{2}}{\varrho_{k}^{2}\left|\ln \varrho_{k}\right|^{2 q}} \eta^{2} d x d t \\
& \leqslant 2 \int_{\tau_{1}}^{\tau_{2}} \int_{\Omega} H_{q}\left(\varrho_{k}\right) \eta \eta_{t} d x d t \\
& +C\left[\left(1+\gamma^{-1}\right)(1+|q|)^{2} \int_{\tau_{1}}^{\tau_{2}} \int_{\Omega}\left(\eta^{2}+|\nabla \eta|^{2}\right)\left|\ln \varrho_{k}\right|^{2-2 q} d x d t\right. \\
& \left.+\gamma^{-1}\|\varrho\|_{L^{\infty}} \int_{\tau_{1}}^{\tau_{2}} \int_{\Omega} e^{\gamma|b|^{2}} \varrho \eta^{2} d x d t\right] .
\end{aligned}
$$


В случае $q=1$, когда $\eta(x, t)=\eta_{0}(x)$ при $t \in\left[\tau_{1}, \tau_{2}\right]$, мы имеем $\partial_{t} \eta=0$ на $\left[\tau_{1}, \tau_{2}\right]$ и $N(k)=0$. Доказательство закончено.

Следующие две леммы можно найти в [14], [15] или [16].

Пусть $V^{2}(U \times J)$ - пространство функций $u \in \mathbf{H}^{2,1}(U \times J)$ с конечной нормой

$$
\|u\|_{V^{2}(U \times J)}=\|u\|_{\mathbf{H}^{2,1}(U \times J)}+\sup _{t \in J}\|u(\cdot, t)\|_{L^{2}(U)} .
$$

Лемма 3.3. Пусть $d>2$. Предположим, ито $v \in V^{2}(\Omega \times J) u \partial_{\Omega s}$ почти всякого $t \in J$ функиия $x \rightarrow v(x, t)$ имеет компактный носитель в $\Omega$. Тогда

$$
\|v\|_{L^{2(d+2) / d}(\Omega \times J)} \leqslant C\|v\|_{V^{2}(\Omega \times J)},
$$

где $C$ зависит только от $d$ и объема $|\Omega|$ множества $\Omega$.

Рассморим куб $K(r)=K(y, r)$ и зададим функцию $\psi_{K}$ следующим образом:

$$
\psi_{K}(x):=\prod_{i=1}^{d} \chi_{i}\left(x_{i}\right), \quad x=\left(x_{1}, \ldots, x_{d}\right),
$$

где $\chi_{i} \in C^{1}\left(\left[y_{i}-2 r, y_{i}+2 r\right]\right), \chi_{i}\left(x_{i}\right)=1$ при $\left|x_{i}-y_{i}\right| \leqslant r$ и $\chi_{i}\left(x_{i}\right)=0$ при $\left|x_{i}-y_{i}\right| \geqslant 2 r$. Пусть также $0 \leqslant \psi_{K} \leqslant 1$ и $\left|\nabla \psi_{K}\right| \leqslant c r^{-1}$ с некоторым положительным числом $c$.

Лемма 3.4. Пусть $v \in V^{2}\left(K(R) \times\left(T_{0}, T_{1}\right)\right)$ и для всякой функиии $\psi \in C_{0}^{1}(K(R))$ функиия

$$
t \longmapsto \int_{K(R)} v(x, t) \psi^{2}(x) d x
$$

абсолютно непрерывна. Предположим, ито существуют такие неотрииательная функиия $g \in L^{1}\left(T_{0}, T_{1}\right)$ и постояннье $A_{1}, A_{2}>0$, ито для всякого куба $K(r) \subset K(R)$ и почти всех $t \in\left(T_{0}, T_{1}\right)$ имеем

$$
\frac{d}{d t} \int_{K(2 r)} v(x, t) \psi_{K}^{2}(x) d x+A_{1} \int_{K(2 r)}|\nabla v|^{2} \psi_{K}^{2} d x \leqslant A_{2} g(t)|K(r)|^{1-2 / d} .
$$

Тогда при $T_{0}<s_{1}<s_{2}<t_{1}<t_{2}<T_{1}$ существуют иисла $\lambda>0$ и $\sigma>0$, зависяиие от $s_{1}, s_{2}, t_{1}, t_{2}, d, A_{1}, A_{2} u\|g\|_{L^{1}\left(T_{0}, T_{1}\right)}$, такие, что

$$
\int_{s_{1}}^{s_{2}} \int_{K(R)} e^{-\lambda v} d x d t \int_{t_{1}}^{t_{2}} \int_{K(R)} e^{\lambda v} d x d t \leqslant \sigma .
$$

Зафиксируем куб $K(R)=K(y, R)$ и числа $s_{1}<s_{2}<t_{1}<t_{2}$ такие, что $s_{1}, s_{2}, t_{1}, t_{2} \in\left[T_{0}, T_{1}\right]$, где $\left[T_{0}, T_{1}\right] \subset(0,1)$.

Положим $Q^{-}:=K(R) \times\left[s_{1}, s_{2}\right]$ и $Q^{+}:=K(R) \times\left[t_{1}, t_{2}\right]$.

Следуюшая теорема - основной результат работы. 
Теорема 3.2. Пусть $\mu=\varrho d x d t-$ решение уравнения (1.1), причем коэффиииенть $a^{i j}, b^{i}$ удовлетворяют условиям (C1), (C2) и (C3). Пусть $\operatorname{ess} \sup _{(x, t) \in Q^{-}} \varrho(x, t)>0$. Тогда $|\ln \varrho| \in L^{\infty}\left(Q^{+}\right)$.

Д о к а з а т ел ь с т в о. Для $n \geqslant 0$ пусть $K_{n}:=K\left(R+2^{-n} R\right)$, $\tau_{n}=t_{1}-\left(t_{1}-s_{2}\right)^{-1} 2^{-n-1}$. Положим $\kappa:=\kappa\left(K(R) \times\left[T_{0}, T_{1}\right]\right), m:=m(K(R) \times$ $\left.\left[T_{0}, T_{1}\right]\right)$.

1. Покажем, что при некотором $\lambda>0$ функции $\left|\ln \varrho_{k}\right|^{\lambda}$, где $\varrho_{k}=$ $\varrho+k^{-1}$, имеют равномерно ограниченные по $k$ интегралы. Положим $f\left(\varrho_{k}\right)=\ln \left|\ln \varrho_{k}\right|$. Согласно лемме 3.2 с $q=1$, при $T_{0}<s<t<T_{1}$ имеет место оценка

$$
\begin{aligned}
& \int_{K_{0}}\left[f\left(\varrho_{k}(x, t)\right) \eta^{2}(x)-f\left(\varrho_{k}(x, s)\right) \eta^{2}(x)\right] d x+\frac{m}{3} \int_{s}^{t} \int_{K_{0}}\left|\nabla f\left(\varrho_{k}\right)\right|^{2} \eta^{2} d x d \tau \\
& \leqslant C\left[\left(1+\gamma^{-1}\right) \int_{s}^{t} \int_{K_{0}}\left(\eta^{2}+|\nabla \eta|^{2}\right) d x d \tau\right. \\
& \left.\quad+\gamma^{-1}\|\varrho\|_{L^{\infty}} \int_{s}^{t} \int_{K_{0}} e^{\gamma|b|^{2}} \varrho \eta^{2} d x d \tau\right]
\end{aligned}
$$

если $\eta \in C_{0}^{\infty}\left(K_{0} \times\left(T_{0}, T_{1}\right)\right)$ и $\eta(x, \tau)=\eta_{0}(x)$ при $\tau \in[s, t]$. Пусть $d \gamma=\kappa$. Согласно неравенству Гёльдера,

$$
\int_{K_{0}} e^{\gamma|b|} \varrho \eta^{2} d x \leqslant\left(\int_{K_{0}} e^{\kappa|b|} \varrho \eta^{2} d x\right)^{1 / d}\left(\int_{K_{0}} \varrho \eta^{2} d x\right)^{(d-1) / d} .
$$

Положим

$$
g(t):=1+\left(\int_{K_{0}} e^{\kappa|b(x, t)|} \varrho(x, t) d x\right)^{1 / d} .
$$

Применив неравенство Гёльдера, легко видеть, что $g \in L^{1}\left(T_{0}, T_{1}\right)$. Согласно равенству (3.5), функция

$$
t \longmapsto \int_{K_{0}} f\left(\varrho_{k}(x, t)\right) \eta_{0}^{2}(x) d x
$$

абсолютно непрерывна на всяком внутреннем подотрезке в $\left[T_{0}, T_{1}\right]$. Для произвольного куба $K(r)$ такого, что $K(2 r) \subset K_{0}$, выберем $\eta$ так, чтобы при $\tau \in[s, t]$ было выполнено равенство $\eta(x, \tau)=\eta_{0}(x):=\psi_{K}(x)$, где функция $\psi_{K}$ определена посредством (3.6). Ввиду (3.7) существуют такие числа $A_{1}, A_{2}$, что

$$
\begin{aligned}
& \frac{d}{d t} \int_{K(2 r)} f\left(\varrho_{k}(x, t)\right) \psi_{K}^{2}(x) d x+A_{1} \int_{K(2 r)}\left|\nabla f\left(\varrho_{k}(x, t)\right)\right| \psi_{K}^{2}(x) d x \\
& \quad \leqslant A_{2} g(t)|K(r)|^{1-2 / d} .
\end{aligned}
$$

Это следует из того, что (3.7) верно для всех пар $s, t$ c $s<t$. Итак, предположения леммы 3.4 выполнены. Значит, существуют числа $\lambda, \sigma>0$, 
не зависящие от $k$, такие, что

$$
\int_{s_{1}}^{s_{2}} \int_{K_{0}} e^{-\lambda f\left(\varrho_{k}\right)} d x d t \int_{\tau_{0}}^{t_{2}} \int_{K_{0}} e^{\lambda f\left(\varrho_{k}\right)} d x d t \leqslant \sigma .
$$

Tак как $\operatorname{ess} \sup _{(x, t) \in Q^{-}} \varrho(x, t)>0$, то первый сомножитель слева отличен от нуля. Поэтому, с учетом оценки $0<f\left(\varrho_{k}\right)<f(\varrho)$, получаем, что найдется число $C_{1}>0$, не зависящее от $k$, для которого

$$
\int_{\tau_{0}}^{t_{2}} \int_{K_{0}} e^{\lambda f\left(\varrho_{k}\right)} d x d t \leqslant \sigma\left(\int_{s_{1}}^{s_{2}} \int_{K_{0}} e^{-\lambda f(e)} d x d t\right)^{-1} \leqslant C_{1} .
$$

Поскольку $f\left(\varrho_{k}\right)=\ln \left|\ln \varrho_{k}\right|$, имеем

$$
\int_{\tau_{0}}^{t_{2}} \int_{K_{0}}\left|\ln \varrho_{k}\right|^{\lambda} d x d t \leqslant C_{1}
$$

2. Покажем, что нормы $\|\ln \varrho\|_{L^{p}}$ равномерно ограничены по $p \in$ $[1,+\infty)$. Положим $f\left(\varrho_{k}\right):=\left|\ln \varrho_{k}\right|^{1-q}$. Пусть $\eta \in C_{0}^{1}\left(K_{0} \times\left(T_{0}, T_{1}\right)\right)$. По лемме 3.2 с $\gamma=\kappa$ при $\tau_{0}<s<t<t_{2}$ мы имеем

$$
\begin{aligned}
& \frac{1}{2-2 q} \int_{K_{0}}\left[f\left(\varrho_{k}\right)^{2} \eta^{2}(x, t)-f\left(\varrho_{k}\right)^{2} \eta^{2}(x, s)\right] d x \\
& \quad+\frac{m}{3(1-q)^{2}} \int_{s}^{t} \int_{K_{0}}\left|\nabla\left(f\left(\varrho_{k}\right)\right)\right|^{2} \eta^{2} d x d \tau \\
& \leqslant 2 \int_{s}^{t} \int_{K_{0}}\left|\eta \| \partial_{t} \eta\right| f\left(\varrho_{k}\right)^{2} d x d \tau \\
& \quad+C\left[\left(1+\delta^{-1}\right)(1+|q|)^{2} \int_{s}^{t} \int_{K_{0}}\left(\eta^{2}+|\nabla \eta|^{2}\right) f\left(\varrho_{k}\right)^{2} d x d \tau\right. \\
& \left.\quad+\delta^{-1}\|\varrho\|_{L^{\infty}} \int_{s}^{t} \int_{K_{0}} e^{\kappa|b|^{2}} \varrho \eta^{2} d x d \tau\right] .
\end{aligned}
$$

Положим

$$
\Lambda:=\int_{T_{0}}^{T_{1}} \int_{K_{0}} e^{\kappa|b|^{2}} \varrho d x d t
$$

Пусть $\eta_{n}$ - непрерывно дифференцируемая функция на $\mathbf{R}^{d} \times \mathbf{R}^{1}$, причем

$$
\begin{array}{lll}
\eta_{n}(x, t)=1 & \text { при } & (x, t) \in K_{n+1} \times\left(\tau_{n+1}, t_{2}\right), \\
\eta_{n}(x, t)=0 & \text { при } & (x, t) \notin K_{n} \times\left(\tau_{n}, T_{1}\right),
\end{array}
$$

а также $|\eta(x, t)| \leqslant 1,\left|\partial_{t} \eta_{n}(x, t)\right| \leqslant c_{1} 2^{n},\left|\nabla_{x} \eta_{n}(x, t)\right| \leqslant c_{2} 2^{n}$, где $c_{1}, c_{2}-$ некоторые положительные числа. Тогда, согласно (3.8), мы имеем

$$
\begin{aligned}
\left\|\nabla\left(f\left(\varrho_{k}\right)\right)\right\|_{L^{2}\left(K_{n+1} \times\left(\tau_{n+1}, t_{2}\right)\right)}^{2} & \leqslant C_{2}(1+|q|)^{4} 4^{n}\left(\left\|f\left(\varrho_{k}\right)\right\|_{L^{2}\left(K_{n} \times\left(\tau_{n}, t_{2}\right)\right)}^{2}+\Lambda\right), \\
\sup _{t \in\left(\tau_{n+1}, t_{2}\right)}\left\|f\left(\varrho_{k}(\cdot, t)\right)\right\|_{L^{2}\left(K_{n+1}\right)}^{2} & \leqslant C_{2}(1+|q|)^{3} 4^{n}\left(\left\|f\left(\varrho_{k}\right)\right\|_{L^{2}\left(K_{n} \times\left(\tau_{n}, t_{2}\right)\right)}^{2}+\Lambda\right),
\end{aligned}
$$


где число $C_{2}$ не зависит от $n$ и $k$. Значит, по лемме 3.3 сушествует такое число $C_{3}$, не зависящее от $n$ и $k$, что

$$
\left\|f\left(\varrho_{k}\right)\right\|_{L^{2(d+2) / d}\left(K_{n+1} \times\left(\tau_{n+1}, t_{2}\right)\right)}^{2} \leqslant C_{3}(1+|q|)^{4} 4^{n}\left(\left\|f\left(\varrho_{k}\right)\right\|_{L^{2}\left(K_{n} \times\left(\tau_{n}, t_{2}\right)\right)}^{2}+\Lambda\right) .
$$

Пусть $p_{0}=\lambda, s=(d+2) d^{-1}>1, p_{n}=s^{n} \lambda$. Тогда $p_{n+1}=s p_{n}$ и $p_{n} \rightarrow \infty$. Подставляя в $f\left(\varrho_{k}\right)=\left|\ln \varrho_{k}\right|^{1-q}$ числа $q_{n}=1-p_{n} / 2$, получаем

$$
\left\|\ln \varrho_{k}\right\|_{L^{p_{n+1}}\left(K_{n+1} \times\left(\tau_{n+1}, t_{2}\right)\right)}^{p_{n}} \leqslant C_{3}\left(1+p_{n}\right)^{4} 4^{n}\left(\left\|\ln \varrho_{k}\right\|_{L^{p_{n}}\left(K_{n} \times\left(\tau_{n}, t_{2}\right)\right)}^{p_{n}}+\Lambda\right) .
$$

Для каждого фиксированного $n$, начиная с $n=0$, мы применим теорему Фату при $k \rightarrow \infty$. Это дает такое число $C_{4}>1$, не зависящее от $n$, что

$$
\|\ln \varrho\|_{L^{p_{n+1}}\left(K_{n+1} \times\left(\tau_{n+1}, t_{2}\right)\right)}^{p_{n}} \leqslant C_{4}^{n}\left(\|\ln \varrho\|_{L^{p_{n}}\left(K_{n} \times\left(\tau_{n}, t_{2}\right)\right)}^{p_{n}}+1\right) .
$$

Пусть $Z_{n}=\max \left\{\|\ln \varrho\|_{L^{p_{n}}\left(K_{n} \times\left(\tau_{n}, t_{2}\right)\right)}, 1\right\}$. Тогда $Z_{n+1} \leqslant\left(2 C_{4}\right)^{\lambda^{-1} n s^{-n}} Z_{n}$, откуда

$$
Z_{n} \leqslant\left(2 C_{4}\right)^{\lambda^{-1} \sum_{n} n s^{-n}} Z_{0}
$$

Итак, последовательность $\left\{Z_{n}\right\}$ ограничена, причем существует такое число $C_{5}$, не зависяшее от $n$, что $\|\ln \varrho\|_{L^{p_{n}}\left(K_{n} \times\left(\tau_{n}, t_{2}\right)\right)} \leqslant C_{5}$. Значит, $|\ln \varrho| \in L^{\infty}\left(K_{0} \times\left(t_{1}, t_{2}\right)\right)$. Теорема доказана.

Следствие 3.1. Пусть $\mu=\varrho d x d t$ - неотрицательное решение уравнения (1.1), где коэффициенты $a^{i j} u b^{i}$ удовлетворяют условиям (C1), (C2) $u$ (C3). Пусть $0<\tau<1$, и пусть

$$
\operatorname{esssup}_{(x, t) \in \mathbf{R}^{d} \times(0, \tau)} \varrho(x, t)>0
$$

Тогда мера $\mu$ имеет непрерывную плотность $\varrho$, которая строго положительна на $\mathbf{R}^{d} \times(\tau, 1)$.

Отметим, что условие $\operatorname{ess} \sup _{(x, t) \in \mathbf{R}^{d} \times(0, \tau)} \varrho(x, t)>0$ тривиальным образом выполнено, если при каждом $t>0$ функция $x \mapsto \varrho(x, t)$ является вероятностной плотностью, как это происходит в применениях к переходным вероятностям. Однако в общем случае это условие нельзя заменить предположением, что мера $\mu$ неотрицательна и не равна нулю тождественно на $\mathbf{R}^{d} \times(0, \tau)$ (как в эллиптическом случае). Это видно из следуюшего простого примера. Пусть $d=1$ и $a \in(0,1)$. Положим $\varrho(x, t)=\exp \left((a-t)^{-1} \exp (x)\right)$ при $t>a$ и $\varrho(x, t)=0$ при $t \leqslant a$. Тогда мера $\mu$ с плотностью $\varrho$ на $\mathbf{R}^{1} \times(0,1)$ удовлетворяет уравнению $\mathscr{L}^{*} \mu=0$, где $a^{11}(x)=e^{-x}$ и $b=0$ (которое в терминах $\varrho$ имеет вид $\left.\partial_{t} \varrho=\partial_{x}\left(a^{11} \partial_{x} \varrho\right)\right)$, но это решение равно нулю при $t \leqslant a$. 
3 а м е ч а н и е 3.2. Теорема 3.1 и следствие 3.1 остаются верными с похожими доказательствами, если оператор $\mathscr{L}$ имеет вид

$$
\begin{aligned}
\mathscr{L} u(x, t):= & \partial_{t} u(x, t)+\partial_{x_{i}}\left(a^{i j}(x, t) \partial_{x_{j}} u(x, t)\right) \\
& +b^{i}(x, t) \partial_{x_{i}} u(x, t)+\Theta(x, t) u(x, t),
\end{aligned}
$$

где $a^{i j}$ и $b^{i}$ удовлетворяют условиям (C1), (С2) и (С2), $\Theta \in L_{\mathrm{loc}}^{p}(\mu)$, причем существует такая измеримая функция $\Psi \geqslant 0$, что $\Theta \geqslant-\Psi$ и $\exp (\Psi) \in L_{\text {loc }}^{1}(\mu)$.

3 а м е ч а н и е 3.3 . Если известно, что мера $\mu$ имеет локально ограниченную плотность $\varrho \in \mathbf{H}_{\text {loc }}^{2,1}\left(\mathbf{R}^{d},(0,1)\right)$, то можно рассмотреть неравенство $\mathscr{L}^{*} \mu \leqslant 0$ вместо уравнения (1.1), где

$$
\begin{aligned}
\mathscr{L} u(x, t):= & \partial_{t} u(x, t)+\partial_{x_{i}}\left(a^{i j}(x, t) \partial_{x_{j}} u(x, t)\right) \\
& +b^{i}(x, t) \partial_{x_{i}} u(x, t)-\Psi(x, t) u(x, t) .
\end{aligned}
$$

Неравенство понимается следуюшим образом:

$$
\int_{0}^{1} \int_{\mathbf{R}^{d}} \mathscr{L} \varphi(x, t) d \mu \leqslant 0
$$

для всякой неотрицательной функции $\varphi \in C_{0}^{\infty}\left(\mathbf{R}^{d} \times(0,1)\right)$. Предположим, что $a^{i j}$ и $b^{i}$ удовлетворяют условиям (C1), (C2) и (C3), $\Psi \geqslant 0$ и $\exp (\Psi) \in L_{\text {loc }}^{1}(\mu)$. Тогда $\varrho$ имеет версию, строго положительную при $t>\tau$, где $\operatorname{ess} \sup _{(x, t) \in \mathbf{R}^{d} \times(0, \tau)} \varrho(x, t)>0$.

Подчеркнем еще раз, что полученные выше результаты применимы к уравнениям с операторами недивергентного вида, надо лишь соответствующие условия экспоненциальной интегрируемости накладывать на новый снос с компонентами $b^{j}-\partial_{x_{i}} a^{i j}$.

Отметим, что наши результаты показывают, что локальная экспоненциальная интегрируемость сноса (с квадратом в параболическом случае) относительно решения приводит к тому, что на самом деле снос оказывается локально экспоненциально интегрируем и относительно меры Лебега (из-за непрерывности и строгой положительности плотности решения). Как уже отмечалось, подобного явления не происходит в случае степенной интегрируемости. Хотя экспоненциальная интегрируемость не является необходимой, ее нельзя существенно ослабить даже в одномерном случае. В работе [17] был исследован следующий вопрос. Пусть дана вероятностная мера $\mu$ на $[0,+\infty)$ с абсолютно непрерывной плотностью $\varrho$, и пусть дана положительная выпуклая функция $\psi$ на $[0,+\infty)$. Когда из интегрируемости $\psi\left(\left|\varrho^{\prime} / \varrho\right|\right)$ относительно $\mu$ следует, что $\varrho(x)>0$ почти всюду на $[0,+\infty)$ ? Этот вопрос имеет прямое отношение к нашей задаче в эллиптическом случае, поскольку мера $\mu$ 
удовлетворяет уравнению (1.1) с $A(x)=1$ и $b(x)=-\varrho^{\prime}(x) / \varrho(x)$, т.е. речь идет в точности о $\mu$-интегрируемости $\psi(|b|)$ в этом весьма специальном частном случае. Оказывается, достаточным условием для справедливости упомянутой импликации является неинтегрируемость на бесконечности функции $x^{-2} \ln \psi(x)$. Более того, эта неинтегрируемость также и необходима для того, чтобы для всякой абсолютно непрерывной вероятностной плотности $\varrho$ из $\mu$-интегрируемости $\psi\left(\left|\varrho^{\prime} / \varrho\right|\right)$ следовала положительность $\varrho$ почти всюду. Например, в качестве $\psi$ можно брать функции $\exp (\kappa x)$ с $\kappa>0$ (как мы делали) или даже $\exp (x / \ln x)$, но не $\exp \left(x /|\ln x|^{2}\right)$.

4. Приложения. Полученные результаты позволяют устанавливать наличие строго положительных непрерывных плотностей конечномерных проекций стационарных распределений и переходных вероятностей бесконечномерных диффузий. Начнем с эллиптического случая. Пусть $\mathbf{R}^{\infty}$ - пространство бесконечных вещественных последовательностей с топологией произведения. Предположим, что даны борелевские функции $B^{i}$ на $\mathbf{R}^{\infty}$ и числа $\alpha_{i}>0$. Мы рассмотрим бесконечномерный эллиптический оператор

$$
L \varphi:=\sum_{i=1}^{\infty}\left[\alpha_{i} \partial_{x_{i}}^{2} \varphi+B^{i} \partial_{x_{i}} \varphi\right]
$$

определенный на гладких функциях от конечного числа переменных. Будем говорить, что борелевская вероятностная мера $\mu$ на $\mathbf{R}^{\infty}$ удовлетворяет слабому эллиптическому уравнению

$$
L^{*} \mu=0,
$$

если для каждого $i \geqslant 1$ имеем $B^{i} \in L^{1}(\mu)$ и

$$
\int_{\mathbf{R} \infty} \sum_{i=1}^{\infty}\left[\alpha_{i} \partial_{x_{i}}^{2} \varphi+B^{i} \partial_{x_{i}} \varphi\right] d \mu=0
$$

при всех функциях $\varphi$ на $\mathbf{R}^{\infty}$ вида $\varphi(x)=\varphi_{0}\left(x_{1}, \ldots, x_{n}\right), \varphi_{0} \in C_{0}^{\infty}\left(\mathbf{R}^{n}\right)$. Указанный ряд представляет собой конечную сумму для таких $\varphi$. Меры, удовлетворяющие уравнению (4.1), называются инфинитезимально инвариантными для оператора $L$, так как при широких предположениях это уравнение выполнено для настоящих инвариантных мер диффузии, порожденной $L$ (конечно, при условии, что такая диффузия существует и имеет стационарные распределения). Преимущество рассмотрения инфинитезимально инвариантных мер заключается в том, что уравнение может иметь смысл и обладать решениями при гораздо более слабых предположениях, чем те, которые нужны для существования ассоциированной диффузии. Это касается даже конечномерного случая. Более 
того, наличие решений эллиптического уравнения часто позволяет построить и диффузию (эти вопросы изучены в работах [19]-[21]).

Зафиксируем $d$ и рассмотрим проекцию $P: \mathbf{R}^{\infty} \rightarrow \mathbf{R}^{d}, P(x)=$ $\left(x_{1}, \ldots, x_{d}\right)$. Пусть $\mu_{P}=\mu \circ P^{-1}-$ соответствующая конечномерная проекция меры $\mu$. Поскольку $B^{i} \in L^{1}(\mu)$, то существует условное математическое ожидание $B_{P}$ отображения $P \circ B$ относительно меры $\mu$ и $\sigma$-алгебры $\sigma_{P}$, порожденной $P$. Тогда $B_{P}(x)=b(P x)$ с некоторым борелевским отображением $b: \mathbf{R}^{d} \rightarrow \mathbf{R}^{d}$. Ясно, что $|b| \in L^{1}\left(\mu_{P}\right)$, ибо $\left|B_{P}\right| \in L^{1}(\mu)$. Мера $\mu_{P}$ удовлетворяет уравнению $L_{P}^{*} \mu=0$, где оператор $L_{P}$ имеет вид

$$
L_{P} u:=\sum_{i=1}^{d} \alpha_{i} \partial_{x_{i}}^{2} u+(b, \nabla u) .
$$

Теорема 4.1. Предположим, что борелевская вероятностная мера $\mu$ удовлетворяет уравнению (4.1) и существуют такие числа $\kappa_{i}>0$, что $\exp \left(\kappa_{i}\left|B^{i}\right|\right) \in L^{1}(\mu)$ для каждого $i \geqslant 1$. Тогда для всякой проекиии $P: \mathbf{R}^{\infty} \rightarrow \mathbf{R}^{d}$ мера $\mu_{P}$ на $\mathbf{R}^{d}$ имеет непрерывную строго положительную плотность.

Д ок аз ат е льст в о. Пусть $\kappa=\min \left(\kappa_{1}, \ldots, \kappa_{d}\right)$. Согласно неравенству Иенсена, имеем $\exp (\kappa|b|) \in L^{1}\left(\mu_{P}\right)$, ибо $\exp \left(\kappa\left|B^{i}\right|\right) \in L^{1}(\mu)$. Значит, применима теорема 3.1 .

В работе [18] с помощью метода функций Ляпунова получены достаточные условия экспоненциальной интегрируемости относительно меры $\mu$, удовлетворяющей уравнению (4.1). Эти условия используют некоторую коэрцитивность сноса. Приведем типичные примеры.

П р и м е $\mathrm{p}$ 4.1. Пусть $q_{n}>0, \sum_{n=1}^{\infty} q_{n}<\infty$ и $X$ - гильбертово пространство последовательностей $x=\left(x_{n}\right)$ с $\|x\|_{0}^{2}:=\sum_{n=1}^{\infty} q_{n} x_{n}^{2}<\infty$. Предположим, что даны функции $B^{n}$ на $X, n \in \mathbf{N}$, которые непрерывны в слабой топологии на шарах, причем при некоторых числах $C_{n}>0$ имеют место оценки

$$
\left|B^{n}(x)\right| \leqslant C_{n}+C_{n}\|x\|_{0}^{2} .
$$

Пусть существует число $C>0$ такое, что

$$
\sum_{n=1}^{\infty} q_{n} x_{n} B^{n}(x) \leqslant C-C\|x\|_{0}^{2}
$$

для всех $x$ вида $x=\left(x_{1}, \ldots, x_{n}, 0, \ldots\right)$. По теореме 5.3 работы [18] на $X$ существует борелевская вероятностная мера $\mu$, удовлетворяющая уравнению (4.1) с $\alpha_{i} \equiv 1$, причем функция $\exp \left(\varepsilon\|x\|_{0}^{2}\right)$ интегрируема относительно $\mu$ при некотором $\varepsilon>0$. Тогда $\exp \left(\varepsilon_{n}\left|B^{n}\right|\right) \in L^{1}(\mu)$ при $\varepsilon_{n}<\varepsilon C_{n}^{-1}$, откуда следует существование непрерывных строго положительных плотностей конечномерных проекций меры $\mu$, порожденных проекциями $x \mapsto\left(x_{1}, \ldots, x_{d}\right)$. 
Если же при некотором $r>1$ известны оценки

$$
\left|B^{n}(x)\right| \leqslant C_{n}+C_{n}\|x\|_{0}^{r}
$$

то достаточно потребовать выполнения неравенства

$$
\sum_{n=1}^{\infty} q_{n} x_{n} B^{n}(x) \leqslant C-C\|x\|_{0}^{r}
$$

на финитных последовательностях.

Аналогичные утверждения справедливы для конечномерных проекций инфинитезимально инвариантных мер для стохастических уравнений типа Бюргерса и Навье-Стокса, построенных в § 7 работы [18], при условии не более чем квадратичного роста нелинейностей. Например, рассмотрим стохастическое уравнение типа Бюргерса

$$
d u(t, x)=\sqrt{2} d W(t, x)+\left[\mathscr{H} u(t, x)-\psi(u(t, x)) \partial_{x} u(t, x)+f(x)\right] d t
$$

в пространстве $X=L^{2}[0,1]$, где $\mathscr{H}$ - оператор Лапласа с нулевыми граничными условиями и собственным базисом $\left\{\eta_{n}\right\}$ с собственными числами $\lambda_{n}, W$ - винеровский процесс в $X$ вида $W(t)=\sum_{n=1}^{\infty} \alpha_{n} w_{n}(t) \eta_{n}$, $\left\{w_{n}\right\}$ - последовательность независимых вещественных винеровских процессов, $\alpha_{n}>0, \sum_{n=1}^{\infty} \alpha_{n}^{2}<\infty, \psi-$ борелевская функция такая, что $|\psi(s)| \leqslant C+C|s|, f \in L^{\infty}[0,1]$. Положим

$$
\begin{gathered}
\Psi(y):=\int_{0}^{y} \psi(s) d s, \\
B^{n}(u)=\lambda_{n} u_{n}+\left(\Psi(u), \eta_{n}^{\prime}\right)_{2}+\left(f, \eta_{n}\right)_{2}, \quad u_{n}:=\left(u, \eta_{n}\right)_{2} .
\end{gathered}
$$

Тогда стационарные распределения указанного стохастического процесса удовлетворяют уравнению $L^{*} \mu=0$ с оператором

$$
L \varphi=\sum_{n=1}^{\infty}\left[\alpha_{n} \partial_{\eta_{n}}^{2} \varphi+B^{n} \partial_{\eta_{n}} \varphi\right]
$$

заданном на функциях вида $\varphi(u)=\varphi_{0}\left(u_{1}, \ldots, u_{n}\right), \varphi_{0} \in C_{0}^{\infty}\left(\mathbf{R}^{n}\right)$, где $\partial_{\eta}$ обозначает частную производную вдоль вектора $\eta$. Как показано в [18], существует борелевская вероятностная мера $\mu$ на $X$, удовлетворяющая уравнению $\mathscr{L}^{*} \mu=0$ в определенном выше смысле. При этом функция $\exp \left(\varepsilon\|x\|_{2}^{2}\right)$ интегрируема относительно $\mu$ при некотором $\varepsilon>0$ (это следует из результатов [18]). Значит, здесь также выполнено условие $\exp \left(\varepsilon_{n}\left|B^{n}\right|\right) \in L^{1}(\mu)$. Аналогично рассматривается стохастическое уравнение типа Навье-Стокса

$$
\begin{aligned}
d u(t, x)= & \sqrt{2} d W(t, x) \\
& +[\mathscr{H} u(t, x)-(u(t, x) \cdot \nabla) u(t, x)+F(x, u(t, x))+\nabla p(t, x)] d t
\end{aligned}
$$


с оператором Лапласа $\mathscr{H}$ на области в $\mathbf{R}^{d}$ и ограниченным непрерывным отображением $F$ (в классическом случае $F$ не зависит от $u=$ $\left.\left(u^{1}, \ldots, u^{d}\right) \in L^{2}\left(D, \mathbf{R}^{d}\right)\right)$. Функции $B^{n}$ здесь имеют вид

$$
B^{n}(u)=\left(\mathscr{H} u, \eta_{n}\right)_{2}+\sum_{j=1}^{d}\left(\partial_{j} u, u^{j} \eta_{n}\right)_{2}+\left(F(\cdot, u), \eta_{n}\right)_{2} .
$$

Соответствуюшая мера $\mu$ строится в пространстве $L^{2}\left(D, \mathbf{R}^{d}\right)$ векторных функций. Отметим, что в работах [23], [24] при отсутствии нелинейности $F$ доказан ряд тонких результатов о плотностях конечномерных проекций стационарных распределений указанного бесконечномерного процесca.

Наконец, еще один близкий пример связан со стохастическим уравнением одномерной диффузионной реакции

$$
d u(t, x)=\left[\partial_{x}^{2} u(t, x)+F(u(t, x))\right] d t+\sqrt{2} d W(t),
$$

где функция $F$ на прямой такова, что $|F(s)| \leqslant C+C s^{2}, s F(s) \leqslant C+\varepsilon s^{2}$ и $\varepsilon>0$ достаточно мало (см. $[18, \S 7])$.

Рассмотрим параболический случай. Аналогом (4.2) для меры $\mu$ на $\mathbf{R}^{\infty} \times(0,1)$ здесь является тождество

$$
\int_{\mathbf{R}^{\infty} \times(0,1)}\left(\partial_{t} \varphi+\sum_{i=1}^{\infty}\left[\alpha_{i} \partial_{x_{i}}^{2} \varphi+B^{i} \partial_{x_{i}} \varphi\right]\right) d \mu=0
$$

для всех функций $\varphi$ вида $\varphi(x, t)=\varphi_{0}\left(x_{1}, \ldots, x_{n}, t\right), \varphi_{0} \in C_{0}^{\infty}\left(\mathbf{R}^{n} \times(0,1)\right)$, причем также требуется $\mu$-интегрируемость коэффициентов $B^{i}: \mathbf{R}^{\infty} \times$ $(0,1) \rightarrow \mathbf{R}^{1}$. В смысле этого тождества и понимается (4.1).

Теорема 4.2. Пусть борелевская вероятностная мера $\mu$ на $\mathbf{R}^{\infty} \times$ $(0,1)$ удовлетворяет уравнению (4.1) и существуют такие числа $\kappa_{i}>0$, что $\exp \left(\kappa_{i}\left|B^{i}\right|^{2}\right) \in L^{1}(\mu)$ для каждого $i \geqslant 1$. Тогда для всякой проекиии $P: \mathbf{R}^{\infty} \times(0,1) \rightarrow \mathbf{R}^{d} \times(0,1), P(x, t)=\left(x_{1}, \ldots, x_{d}, t\right)$, мера $\mu_{P}$ на $\mathbf{R}^{d} \times(0,1)$ имеет непрерывную строго положительную плотность, если $\mu\left(\mathbf{R}^{\infty} \times(0, \tau)\right)>0$ при всех $\tau>0$.

Доказательство аналогично обоснованию предыдущей теоремы. Такие же соображения применимы в следующей более общей ситуации. Предположим, что $X$ - локально выпуклое пространство и для каждого $n$ задана борелевская функция $B^{n}$ на $X \times(0,1)$. Пусть $a^{i j}-$ вешественные числа, причем матрицы $\left(a^{i j}\right)_{i, j \leqslant d}$ положительны. Пусть $l_{i}-$ непрерывные линейные функционалы на $X$ и пусть $h_{j}$ - векторы в $X$ такие, что $l_{i}\left(h_{j}\right)=\delta_{i j}$. Рассмотрим оператор

$$
L \varphi:=\partial_{t} \varphi+\sum_{i, j \geqslant 1} a^{i j} \partial_{h_{i}} \partial_{h_{j}} \varphi+\sum_{i \geqslant 1} B^{i} \partial_{h_{i}} \varphi
$$


определенный на функциях вида $\varphi(x, t)=\varphi_{0}\left(l_{1}(x), \ldots, l_{n}(x), t\right), \varphi_{0} \in$ $C_{0}^{\infty}\left(\mathbf{R}^{n} \times(0,1)\right)$. Тогда для борелевской вероятностной меры $\mu$ на $X \times(0,1)$, для которой $B^{i} \in L^{1}(\mu)$ при всех $i$, уравнение $L^{*} \mu=0$ определяется в том же смысле, что и выше. Для фиксированного числа $d$ пусть $b^{i}$ обозначает условное математическое ожидание $B^{i}$ относительно меры $\mu$ и $\sigma$-алгебры, порожденной $l_{1}, \ldots, l_{d}$. Положим $\pi(x, t):=$ $\left(l_{1}(x), \ldots, l_{d}(x), t\right),(x, t) \in X \times(0,1), \mu_{\pi}:=\mu \circ \pi^{-1}$. Тогда мера $\mu_{\pi}$ на $\mathbf{R}^{d} \times(0,1)$ удовлетворяет параболическому уравнению $L_{\pi}^{*} \mu_{\pi}=0$ с оператором $L_{\pi}$, имеющим матрицу диффузии $\left(a^{i j}\right)_{i, j \leqslant d}$ и снос $b_{\pi}=\left(b^{i}\right)$. Следовательно, приходим к такому утверждению.

Теорема 4.3. Предположим, что для каждого $i$ существует такое число $\kappa_{i}>0$, что $\exp \left(\kappa_{i}\left|B^{i}\right|^{2}\right) \in L^{1}(\mu)$. Тогда мера $\mu_{\pi}$ имеет непрерьвную строго положительную плотность на $\mathbf{R}^{d} \times(0,1)$, если $\mu\left(\mathbf{R}^{\infty} \times(0, \tau)\right)>0$ при всех $\tau>0$.

Отметим, что в предыдущих результатах речь идет о конечномерных проекциях, заданных функционалами вида, упомянутого в условиях (правда, в случае $\mathbf{R}^{\infty}$ всякий непрерывный линейный функционал есть конечная линейная комбинация координатных). В некоторых случаях, как в следуюшем примере, можно от специальных функционалов переходить к общим.

П р и м е р 4.2. Пусть диффузионный процесс $\xi_{t}$ в сепарабельном банаховом пространстве $X$ задан стохастическим дифференциальным уравнением

$$
d \xi_{t}=d W_{t}+b\left(\xi_{t}, t\right) d t
$$

где $W_{t}$ - некоторый винеровский процесс в $X$ (см. [22, гл. 7]), для которого распределение $W_{1}$ положительно на всех шарах, $b: X \times[0,1] \rightarrow$ $X$ - борелевское отображение, причем $\|b(x, t)-b(y, t)\| \leqslant C\|x-y\|$ и $\|b(x, t)\| \leqslant C+C\|x\|$, где $C$ - постоянная. Это уравнение понимается как интегральное:

$$
\xi_{t}(\omega)=\xi_{0}(\omega)+W_{t}(\omega)+\int_{0}^{t} b\left(\xi_{s}(\omega), s\right) d s .
$$

Оно имеет единственное решение, которое строится с помощью теоремы о сжимающем отображении. Предположим, что при некотором $\varepsilon_{0}>0$ функция $\exp \left(\varepsilon_{0}\|x\|^{2}\right)$ интегрируема относительно распределения $\xi_{0}$ (что выполнено, например, если $\xi_{0}$ - неслучайная точка). Используя лемму Гронуолла, получаем

$$
\left\|\xi_{t}(\omega)\right\| \leqslant\left[\left\|\xi_{0}(\omega)\right\|+\left\|W_{t}(\omega)\right\|+C\right] e^{C t}, \quad t \in[0,1] .
$$

Ввиду теоремы Ферника (см. $[22, \S 2.6])$ при некотором $\varepsilon_{1}>0$ случайные величины $\exp \left(\varepsilon_{1}\left\|W_{t}\right\|^{2}\right)$, где $t \in[0,1]$, имеют равномерно ограниченные 
математические ожидания. Поэтому найдется такое $\varepsilon_{2}>0$, что случайные величины $\exp \left(\varepsilon_{2}\left\|\xi_{t}\right\|^{2}\right)$ при $t \in[0,1]$ также имеют равномерно ограниченные математические ожидания. Это означает, что функция $\exp \left(\varepsilon_{2}\|x\|^{2}\right)$ интегрируема относительно меры $P(t, \cdot) d t$ на $X \times[0,1]$, что дает также интегрируемость $\exp \left(C^{-2} \varepsilon_{2}\|b\|^{2}\right)$ относительно этой меры. Предположим, что $l_{1}, \ldots, l_{d} \in X^{*}$ и отображение $\pi=\left(l_{1}, \ldots, l_{d}\right): X \rightarrow \mathbf{R}^{d}$ сюръективно. Положим $P_{t}(B):=\mathbf{P}\left\{\xi_{t} \in B\right\}$ и рассмотрим меру $\mu:=P_{t} d t$ на $X \times(0,1)$. Как и выше, существуют борелевские функции $b^{i}$ на $\mathbf{R}^{d} \times(0,1)$ такие, что $b^{i} \circ \pi$ совпадает с условным математическим ожиданием $l_{i} \circ b$ относительно меры $\mu$ и $\sigma$-алгебры, порожденной $\pi$. Нетрудно проверить, что мера $\mu_{\pi}:=\mu \circ \pi^{-1}$ на $\mathbf{R}^{d} \times(0,1)$ удовлетворяет уравнению (1.1) со сносом $b_{\pi}:=\left(b^{1}, \ldots, b^{d}\right)$ и некоторыми постоянными коэффициентами $a^{i j}$. Пользуясь тем, что распределение $W_{1}$ не сосредоточено на собственном замкнутом подпространстве, можно показать, что матрица $\left(a^{i j}\right)$ строго положительна. Следовательно, согласно теореме 3.2 , мера $\mu_{\pi}$ обладает строго положительной непрерывной плотностью. Это же можно получить непосредственно из теоремы 4.3. Действительно, можно показать, что существуют такие функционалы $f_{i} \in X^{*}$, что $f_{i}\left(W_{t}\right)$ являются независимыми винеровскими процессами, а функционалы $l_{1}, \ldots, l_{d}$ являются линейными комбинациями $f_{1}, \ldots, f_{d}$. Для этого обозначим через $\gamma$ распределение $W_{1}$ в $X$, применим стандартную процедуру ортогонализации к элементам $l_{1}, \ldots, l_{d}$ пространства $L^{2}(\gamma)$ и дополним полученные функционалы $f_{1}, \ldots, f_{d}$ до ортонормированного базиса евклидова пространства $X^{*}$, наделенного скалярным произведением из $L^{2}(\gamma)$ (см. [22, §7.2] о бесконечномерных винеровских процессах). Фактически мы строим другой винеровский процесс $\widetilde{W}_{t}$ вида $\widetilde{W}_{t}=\sum_{i=1}^{\infty} w_{t}^{i} e_{i}$, где $\left\{e_{i}\right\}-$ ортонормированный базис в пространстве Камерона-Мартина меры $\gamma$ и $w_{t}^{i}$ - независимые вещественные винеровские процессы, причем процесс $\widetilde{W}_{t}$ имеет такие же конечномерные распределения, как и $W_{t}$, и $f_{i}\left(e_{j}\right)=\delta_{i j}$. Тогда теорема 4.3 применима к проекциям меры $\mu$, задаваемым функционалами $f_{i}$. Остается заметить, что существует обратимый оператор $S$ на $\mathbf{R}^{d}$, для которого $S \circ\left(f_{1}, \ldots, f_{d}\right)=\left(l_{1}, \ldots, l_{d}\right)$.

Было бы интересно изучить конечномерные проекции меры $\mu$ в теореме 4.3 , соответствуюшие произвольным непрерывным конечномерным операторам, не обязательно порожденным функционалами $l_{i}$.

\section{СПИСОК ЛИТЕРАТУРЫ}

1. Bogachev V.I., Krylov N.V., Röckner M. On regularity of transition probabilities and invariant measures of singular diffusions under minimal conditions. - Comm. Partial Differential Equations, 2001, v. 26, №11-12, p. 2037-2080. 
2. Шапошников С. В. Положительность инвариантных мер диффузионных процессов. - Докл. РАН, 2007, т. 415, № 2, с. 174-179.

3. Metafune G., Pallara D., Rhandi A. Global properties of invariant measures. - J. Funct. Anal., 2005, v. 223, № 2, p. 396-424.

4. Богачёв В.И., Рёкнер М., Шапошников С. В. Глобальная регулярность и оценки решений параболических уравнений. - Теория вероятн. и ее примен., 2005, т. 50, B. 4 , c. $652-674$.

5. Bogachev V.I., Krylov N. V., Röckner M. Elliptic equations for measures: regularity and global bounds of densities. - J. Math. Pures Appl., 2006, v. 85, № 6, p. 743-757.

6. Богачёв В. И., Рёкнер М., Шапошников С. В. Оценки плотностей стационарных распределений и переходных вероятностей диффузионных процессов. - Теория вероятн. и ее примен., 2007, т. 52 , в. 2 , с. 240-270.

7. Богачёв В.И., Рёкнер М. Обобщение теоремы Хасьминского о существовании инвариантных мер для локально интегрируемых сносов. - Теория вероятн. и ее примен., 2000, т. 45, в. 3, с. 417-436.

8. Bogachev V.I., Da Prato G., Röckner M. On parabolic equations for measures. Comm. Partial Differential Equations, 2008, v. 33, № 3, p. 397-418.

9. Богачёв В. И., Рёкнер М., Штаннат В. Единственность решений эллиптических уравнений и единственность инвариантных мер диффузий. - Матем. сб., 2002, т. 193, № 7, c. 3-36.

10. Bogachev V.I., Da Prato G., Röckner M., Stannat W. Uniqueness of solutions to weak parabolic equations for measures. - Bull. London Math. Soc., 2007, v. 39, № 4, p. 631-640.

11. Шапошников С.В. О неединственности решений эллиптических уравнений для вероятностных мер. - Докл. РАН, 2008 (в печати).

12. Krylov N. V. Some properties of traces for stochastic and deterministic parabolic weighted Sobolev spaces. - J. Funct. Anal., 2001, v. 183, № 1, p. 1-41.

13. Krylov N. V. An analytic approach to SPDEs. - Stochastic Partial Differential Equations: Six Perspectives. Ed. by R. Carmona and B. Rozovskii. Providence: Amer. Math. Soc., 1999, p. 185-242.

14. Trudinger N.S. Pointwise estimates and quasilinear parabolic equations. - Comm. Pure Appl. Math., 1968, v. 21, p. 205-226.

15. Aronson D. G., Serrin J. Local behavior of solutions of quasilinear parabolic equations. - Arch. Ration. Mech. Anal., 1967, v. 25, p. 81-122.

16. Moser J. A Harnack inequality for parabolic differential equations. - Comm. Pure Appl. Math., 1964, v. 17, p. 101-134; corrections: ibid., 1967, v. 20, p. 231-236.

17. Scheutzow M., von Weizsäcker $H$. Which moments of a logarithmic derivative imply quasiinvariance? - Doc. Math., 1998, v. 3, p. 261-272.

18. Bogachev V.I., Röckner M. Elliptic equations for measures on infinite-dimensional spaces and applications. - Probab. Theory Related Fields, 2001, v. 120, № 4, p. 445496.

19. Eberle A. Uniqueness and Nonuniqueness of Semigroups Generated by Singular Diffusion Operators. Berlin: Springer, 1999, 262 p. (Lecture Notes in Math., v. 1718.)

20. Stannat $W$. (Nonsymmetric) Dirichlet operators on $L^{1}$ : existence, uniqueness and associated Markov processes. - Ann. Sc. Norm. Super. Pisa Cl. Sci. (4), 1999, v. 28, № 1, p. 99-140.

21. Stannat $W$. Time-dependent diffusion operators on $L^{1}$. - J. Evol. Equ., 2004, v. 4, № 4, p. 463-495.

22. Богачёв В. И. Гауссовские меры. М.: Наука, 1997, 352 с.

23. Agrachev A., Kuksin S., Sarychev A., Shirikyan A. On finite-dimensional projections of distributions for solutions of randomly forced 2D Navier-Stokes equations. - Ann. Inst. H. Poincaré, 2007 , v. 43, № 4, p. 399-415.

24. Shirikyan A. Qualitative properties of stationary measures for three-dimensional Navier-Stokes equations. - J. Funct. Anal., 2007, v. 249, № 2, p. 284-306. 